\title{
On the well-posedness of solutions with finite energy for nonlocal equations of porous medium type
}

Félix del Teso, Jørgen Endal, and Espen R. Jakobsen

Dedicated to Helge Holden, who never stops inspiring us, on the occasion of his 60th birthday.

\begin{abstract}
We study well-posedness and equivalence of different notions of solutions with finite energy for nonlocal porous medium type equations of the form

$$
\partial_{t} u-A \varphi(u)=0 .
$$

These equations are possibly degenerate nonlinear diffusion equations with a general nondecreasing continuous nonlinearity $\varphi$ and the largest class of linear symmetric nonlocal diffusion operators $A$ considered so far. The operators are defined from a bilinear energy form $\mathcal{E}$ and may be degenerate and have some $x$-dependence. The fractional Laplacian, symmetric finite differences, and any generator of symmetric pure jump Lévy processes are included. The main results are (i) an Oleĭnik type uniqueness result for energy solutions; (ii) an existence (and uniqueness) result for distributional solutions with finite energy; and (iii) equivalence between the two notions of solution, and as a consequence, new well-posedness results for both notions of solutions. We also obtain quantitative energy and related $L^{p}$-estimates for distributional solutions. Our uniqueness results are given for a class of functions defined from test functions by completion in a certain topology. We study rigorously several cases where this space coincides with standard function spaces. In particular, for operators comparable to fractional Laplacians, we show that this space is a parabolic homogeneous fractional Sobolev space.
\end{abstract}

2010 Mathematics Subject Classification. 35A02, 35B30, 35D30, 35K55, 35K65, 35R09, 35R11

Keywords. uniqueness, existence, energy solutions, distributional solutions, nonlinear degenerate diffusion, porous medium equation, Stefan problem, fractional Laplacian, nonlocal operators, bilinear forms, Dirichlet forms, homogeneous fractional Sobolev spaces.

\section{Contents}

1 Introduction

2 Main results

2.1 Assumptions . . . . . . . . . . . . . . . . . 5

2.2 Uniqueness results for energy solutions . . . . . . . . . . . 
2.3 Equivalence with distributional solutions and consequences . . 10

2.4 Remarks . . . . . . . . . . . . . . . . . . 13

3 Proof of uniqueness for energy solutions 15

4 Distributional solutions with finite energy 18

4.1 Equivalent notions of solutions . . . . . . . . . . 18

4.2 The approximate problem of (1.3) $-(1.4) \ldots \ldots \ldots$

Acknowledgments 27

A Proof of Theorem 2.6 (a) 27

B On the spaces $\dot{H}^{\frac{\alpha}{2}}\left(\mathbb{R}^{N}\right)$ and $L^{2}\left(0, T ; \dot{H}^{\frac{\alpha}{2}}\left(\mathbb{R}^{N}\right)\right)$

C Proof of Theorem 2.6 (b) 33

D Proof of Lemma 4.3

References

\section{Introduction}

In this paper we study uniqueness and existence of solutions with finite energy of the following two related Cauchy problems of nonlocal porous medium type,

$$
\begin{aligned}
\partial_{t} u-A^{\lambda}[\varphi(u)] & =0 & & \text { in } Q_{T}:=\mathbb{R}^{N} \times(0, T), \\
u(x, 0) & =u_{0}(x) & & \text { on } \mathbb{R}^{N},
\end{aligned}
$$

and

$$
\begin{aligned}
\partial_{t} u-\mathcal{L}^{\mu}[\varphi(u)] & =0 & & \text { in } Q_{T}, \\
u(x, 0) & =u_{0}(x) & & \text { on } \mathbb{R}^{N},
\end{aligned}
$$

where $u=u(x, t)$ is the solution, $T>0, A^{\lambda}$ and $\mathcal{L}^{\mu}$ are nonlocal (convection-) diffusion operators, the nonlinearity $\varphi$ is any continuous nondecreasing function, and $u_{0} \in L^{1} \cap L^{\infty}$. The problems are nonlinear degenerate parabolic and include the fractional porous medium equations [26] where $\mathcal{L}^{\mu}=-(-\Delta)^{\frac{\alpha}{2}}$ and $\varphi(u)=u|u|^{m-1}$ for $\alpha \in(0,2)$ and $m>0$. Included are also Stefan problems, filtration equations, and generalized porous medium equations, see the introductions of 26, 24, 22] for more information.

Both problems are connected to a bilinear energy form defined as

$$
\mathcal{E}_{\lambda}[f, g]:=\frac{1}{2} \iint_{\mathbb{R}^{N} \times \mathbb{R}^{N} \backslash D}(f(y)-f(x))(g(y)-g(x)) \Lambda(\mathrm{d} x, \mathrm{~d} y),
$$


where $D:=\left\{(x, x): x \in \mathbb{R}^{N}\right\}$ is the diagonal and $\Lambda$ is a nonnegative Radon measure on $\mathbb{R}^{N} \times \mathbb{R}^{N} \backslash D$. The operator $A^{\lambda}$ is the generator of $\mathcal{E}_{\lambda}$ defined by

$$
\mathcal{E}_{\lambda}[f, g]=-\int_{\mathbb{R}^{N}} f A^{\lambda}[g] \mathrm{d} x
$$

(see Corollary 1.3.1 in [29]), while $\mathcal{L}^{\mu}=A^{\lambda}$ for the special case where $\Lambda=$ $\mu(x+\mathrm{d} y) \mathrm{d} x$. In general $A^{\lambda}$ is symmetric, $x$-dependent, and has no closed expression, while $\mathcal{L}^{\mu}$ is an $x$-independent operator with integral representation

$$
\mathcal{L}^{\mu}[\phi](x)=\int_{\mathbb{R}^{N} \backslash\{0\}}\left(\phi(x+z)-\phi(x)-z \cdot D \phi(x) \mathbf{1}_{|z| \leq 1}\right) \mu(\mathrm{d} z),
$$

where $D$ is the gradient, $\mathbf{1}_{|z| \leq 1}$ an indicator function, and $\mu$ a symmetric (even) nonpositive Lévy measure satisfying $\int|z|^{2} \wedge 1 \mu(\mathrm{d} z)<\infty$. The operator $\mathcal{L}^{\mu}$ is nonnegative and symmetric and the fractional Laplacian is an example.

A first warning is that $A^{\lambda}$ is not a pure diffusion operator in general: Under density and symmetry assumptions on $\Lambda, A^{\lambda}$ will have an integral representation like (1.7) with $x$-depending $\mu$ plus an additional drift term! A second warning is that the $x$-dependence in $A^{\lambda}$ is restricted, e.g. $-a(x)(-\Delta)^{\frac{\alpha}{2}}$ is not covered! We refer to Section 2.17 for precise assumptions and to Section 2.4 for a discussion and examples of $A^{\lambda}$.

The inspiration for this work were the two recent papers [24] and 22] which contain well-posedness results for energy (or weak) solutions of (1.1)-(1.2) and distributional (or very weak) solutions of (1.3)-(1.4) respectively. These very general results requires different techniques and formulations. The uniqueness argument of [22] is based on a complicated resolvent approximation procedure of Brézis and Crandall [18], while in [24] it is based on an easier and more direct argument by Ole⿳ninik et al. 32 .

The first part of this paper is devoted to Oleinnik type uniqueness arguments for (1.1)-(1.2). We try to push this argument as far as possible, and in the process we extend some of the results and arguments of [24]. E.g., we remove absolute continuity, symmetry, and comparability assumptions. We also discuss the applicability and limitations of the method. Our uniqueness results are given for a class of functions defined from test functions by completion in a certain topology. We study rigorously several cases where this space coincides with standard function spaces. In particular, for operators (globally) comparable to fractional Laplacians, we show that this space is a parabolic homogeneous fractional Sobolev space. In an appendix we also provide rigorous definitions and results of these spaces, some of which we were not able to find in the literature.

In the second part of the paper we study the equivalence between energy and distributional formulations in the setting of (1.3)-(1.4). A main result is a new existence result for distributional solutions with finite energy. This existence result and the uniqueness result of [22] is then transported from 
distributional solutions to energy solutions by equivalence, while the Oleinnik uniqueness results of the first part is transported in the other direction. These result are all either new, or for the Oleĭnik results, represent a much simpler approach to obtaining uniqueness compared to [22]. At the end, we give several new quantitative energy and related $L^{p}$-estimates for distributional solutions.

The type of bilinear form defined in (1.5) plays a central role in probability theory. It is associated with a Dirichlet form and a corresponding symmetric Markov process, see e.g. [29] for a general theory. The type of "nonlocal" bilinear form we consider here is similar to those studied in e.g. [35, 5]. In the linear case $(\varphi(u)=u)$, equations (1.1) and (1.3) are (at least formally) Kolmogorov equations for the transition probability densities of the corresponding Markov processes (see e.g. Section 3.5.3 in [4]).

Let us now give a brief summary of previous works on (1.1)-1.2 and (1.3)-(1.4). We focus first on the $x$-dependent equation (1.1). In the linear case there is a large amount of literature. Some of the main trends in the more PDE oriented community are described in the two surveys [31, 37] (along with extensions to other types of nonlinear equations). When $\varphi$ is nonlinear, we are not aware of any other result than the ones presented in 24]. There the authors consider operators $A^{\lambda}$ where the densities of the measures are comparable to the density of the fractional Laplacian. Existence and uniqueness is discussed in the first part, but the main focus of the paper is to prove continuity/regularity and long time asymptotics for energy solutions.

There is a vast literature on special cases of (1.3)-(1.4). In the linear fractional case $\partial_{t} u+(-\triangle)^{\frac{\alpha}{2}} u=0$ for $\alpha \in(0,2)$, we have well-posedness even for measure data and solutions growing at infinity [6, 13. If we replace $(-\triangle)^{\frac{\alpha}{2}}$ by an operator $\mathcal{L}$ whose measure has integrable density, well-posedness results can be found in [17]. In the case of the fractional porous medium equation (see above), existence, uniqueness and a priori estimates are proven for (strong) $L^{1}$-energy solutions in [25, 26]. We also mention that there are results for that equation in weighted $L^{1}$-spaces [14, with logarithmic diffusion $(\varphi(u)=\log (1+u))$ 27], singular or ultra fast diffusions [11, weighted equations with measure data [30, and problems on bounded domains [12, 15, 16. There are other ways to investigate these equations: In [10, 19, 38, 9, 40, the authors consider a so-called porous medium equation with fractional pressure, and in 3 they consider bounded diffusion operators that can be represented by nonsingular integral operators on the form (1.7). Finally, we mention that in the presence of (nonlinear) convection in (1.3)-(1.4), additional entropy conditions are needed to have uniqueness [1, 20, 21]; a counterexample for uniqueness of distributional solutions is given in 2].

Outline. In Section 2 we state the assumptions and present and discuss our main results. The main uniqueness result is proven in Section 3, Properties such as equivalence of distributional and energy solutions, existence of distri- 
butional solutions with finite energy, and energy and $L^{p}$-estimates are finally proven in Section 4 . In Appendices $\mathrm{A}, \mathrm{B}$ and $\mathrm{C}$ we give rigorous results on the Sobolev spaces we use in this paper along with the proofs of characterizations of the uniqueness function class in terms of common function spaces.

Notation. We use the same notation as in 22 except for the ones we explicitly mention here: The (Borel) measure $\mu$ is said to be even if $\mu(B)=\mu(-B)$ for all Borel sets $B$. We say that the (Borel) measure $\Lambda(\mathrm{d} x, \mathrm{~d} y)$ is symmetric if $\Lambda(\mathrm{d} x, \mathrm{~d} y)=\Lambda(\mathrm{d} y, \mathrm{~d} x)$. A kernel $\lambda(x, \mathrm{~d} y)$ on $\mathbb{R}^{N} \times \mathcal{B}\left(\mathbb{R}^{N} \backslash\{x\}\right)$ satisfies: (i) $B \mapsto \lambda(x, B)$ is a positive measure on $\mathcal{B}\left(\mathbb{R}^{N} \backslash\{x\}\right)$ for each fixed $x \in \mathbb{R}^{N}$; and (ii) $x \mapsto \lambda(x, B)$ is a Borel measurable function for every $B \in \mathcal{B}\left(\mathbb{R}^{N} \backslash\{x\}\right)$. An operator $L$ is symmetric on $L^{2}$ if $(u, L v)_{L^{2}}=(L u, v)_{L^{2}}$. From the bilinear form $\mathcal{E}_{\lambda}$ defined in (1.5) we define a seminorm (the energy) and a space,

$$
|f|_{E_{\lambda}}^{2}:=\mathcal{E}_{\lambda}[f, f] \text { and } E_{\lambda}\left(\mathbb{R}^{N}\right):=\left\{f \text { is measurable }:|f|_{E_{\lambda}}<\infty\right\},
$$

and the related parabolic (energy) seminorm and space,

$$
\begin{aligned}
& |f|_{T, E_{\lambda}}^{2}:=\int_{0}^{T}|f(\cdot, t)|_{E_{\lambda}}^{2} \mathrm{~d} t, \\
& L^{2}\left(0, T ; E_{\lambda}\left(\mathbb{R}^{N}\right)\right):=\left\{f: Q_{T} \rightarrow \mathbb{R} \text { is measurable }:|f|_{T, E_{\lambda}}<\infty\right\} .
\end{aligned}
$$

The Cauchy-Schwartz inequality holds in this setting (cf. Lemma 3.1):

$$
\left|\int_{0}^{T} \mathcal{E}_{\lambda}[f(\cdot, t), g(\cdot, t)] \mathrm{d} t\right| \leq|f|_{T, E_{\lambda}}|g|_{T, E_{\lambda}} .
$$

\section{Main results}

In this section we give the assumptions, main results, and a discussion of these. There are two sections with results. Section 2.2 contains a sequence of uniqueness results for energy solutions of (1.1)-(1.2), while Section 2.3 contains results about (1.3)-(1.4). There we prove the equivalence of energy and distributional solutions with finite energy, the existence of the latter type of solutions, and transport uniqueness and existence results between the two formulations. The results we obtain are either new or represent a much more efficient way to obtain such results compared to previous arguments.

2.1. Assumptions. We start by the bilinear form $\mathcal{E}_{\lambda}$ defined in (1.5). To have a more practical formulation of the assumptions, we first rewrite (1.5): We assume that $\Lambda$ has as kernel $\tilde{\lambda} \geq 0$ with respect to $\mathrm{d} x, \Lambda(\mathrm{d} x, \mathrm{~d} y)=\tilde{\lambda}(x, \mathrm{~d} y) \mathrm{d} x$, change variables $y \rightarrow x+z$, and set $\lambda(x, \mathrm{~d} z):=\tilde{\lambda}(x, x+\mathrm{d} z)$ to obtain

$$
\mathcal{E}_{\lambda}[f, g]=\frac{1}{2} \int_{\mathbb{R}^{N}} \int_{|z|>0}(f(x+z)-f(x))(g(x+z)-g(x)) \lambda(x, \mathrm{~d} z) \mathrm{d} x .
$$


Our assumptions on $\mathcal{E}_{\lambda}$ can then be formulated as follows:

$\left(\mathbf{A}_{\lambda 0}\right) \Lambda$ has as kernel $\tilde{\lambda} \geq 0$ on $\mathbb{R}^{N} \times \mathcal{B}\left(\mathbb{R}^{N} \backslash\{x\}\right)$,

$$
\Lambda(\mathrm{d} x, \mathrm{~d} y)=\tilde{\lambda}(x, \mathrm{~d} y) \mathrm{d} x .
$$

$\left(\mathbf{A}_{\lambda 1}\right)$ The translated kernel $\lambda(x, \mathrm{~d} z):=\tilde{\lambda}(x, x+\mathrm{d} z)$ satisfies

(i) $\Sigma_{\lambda}(x):=\int_{0<|z| \leq 1}|z|^{2} \lambda(x, \mathrm{~d} z) \in L_{\text {loc }}^{1}\left(\mathbb{R}^{N}\right)$; and

(ii) $\Pi_{\lambda}(x):=\int_{|z|>1} \lambda(x, \mathrm{~d} z) \in L_{\mathrm{loc}}^{1}\left(\mathbb{R}^{N}\right)$.

$\left(\mathbf{A}_{\lambda 2}\right) \Lambda$ is symmetric,

$$
\int_{A} \int_{B} \Lambda(\mathrm{d} x, \mathrm{~d} y)=\int_{B} \int_{A} \Lambda(\mathrm{d} x, \mathrm{~d} y) \text { for all Borel } A \times B \subset \mathbb{R}^{N} \times \mathbb{R}^{N} \backslash D .
$$

In some results, we need to strengthen assumption $\left(\mathbf{A}_{\lambda 1}\right)$.

$\left(\mathbf{A}_{\lambda 1}{ }^{\prime}\right)$ Assumption $\left(\mathbf{A}_{\lambda 1}\right)$ holds and in addition

(i) $\Pi_{\lambda} \in L^{\infty}\left(\mathbb{R}^{N}\right)$; and

(ii) $\lambda(x, \mathrm{~d} z)$ is locally shift-bounded: For some constant $C>0$,

$\lambda(x+h, B) \leq C \lambda(x, B) \quad$ for all $\quad x, h \in \mathbb{R}^{N},|h| \leq 1$, Borel $B \subset B(0,1) \backslash\{0\}$.

$\left(\mathbf{A}_{\lambda 1} "\right)$ Assumption $\left(\mathbf{A}_{\lambda 1}\right)$ holds and in addition

$$
m \mu_{\alpha}(\mathrm{d} z) \leq \lambda(x, \mathrm{~d} z) \leq M \mu_{\alpha}(\mathrm{d} z) \quad \text { where } \quad \mu_{\alpha}(\mathrm{d} z)=\frac{c_{N, \alpha} \mathrm{d} z}{|z|^{N+\alpha}},
$$

for some $0<m \leq M, \alpha \in(0,2)$, and every $x \in \mathbb{R}^{N}$.

The remaining assumptions we will use in this paper are given below.

$\left(\mathbf{A}_{\mu}\right) \quad \mu \geq 0$ is an even Radon measure on $\mathbb{R}^{N} \backslash\{0\}$ satisfying

$$
\int_{|z| \leq 1}|z|^{2} \mu(\mathrm{d} z)+\int_{|z|>1} 1 \mu(\mathrm{d} z)<\infty .
$$

$\left(\mathbf{A}_{\varphi}\right) \varphi: \mathbb{R} \rightarrow \mathbb{R}$ is continuous and nondecreasing.

$\left(\mathbf{A}_{u_{0}}\right) u_{0} \in L^{1}\left(\mathbb{R}^{N}\right) \cap L^{\infty}\left(\mathbb{R}^{N}\right)$.

Remark 2.1. (a) By $\left(\mathrm{A}_{\lambda 0}\right)$ and $\left(\mathrm{A}_{\lambda 1}\right), \mathcal{E}_{\lambda}[\cdot, \cdot]$ is well-defined on $C_{\mathrm{c}}^{\infty}\left(\mathbb{R}^{N}\right)$, nonnegative and symmetric,

$$
\mathcal{E}_{\lambda}[f, f] \geq 0 \quad \text { and } \quad \mathcal{E}_{\lambda}[f, g]=\mathcal{E}_{\lambda}[g, f] \quad \text { for } \quad f, g \in C_{\mathrm{c}}^{\infty}\left(\mathbb{R}^{N}\right) .
$$

Moreover, by Example 1.2.4 in [29], $\left(\mathcal{E}_{\lambda}, C_{\mathrm{c}}^{\infty}\left(\mathbb{R}^{N}\right)\right)$ is a closable Markovian form on $L^{2}\left(\mathbb{R}^{N}\right)$ and its closure a regular Dirichlet form. 
(b) It is easy to check that $\left(\mathrm{A}_{\lambda 1}{ }^{\prime \prime}\right) \Rightarrow\left(\mathrm{A}_{\lambda 1}{ }^{\prime}\right) \Rightarrow\left(\mathrm{A}_{\lambda 1}\right)$, see also the remarks on locally shift-bounded kernels in Section 2.4 Assumption $\left(\mathrm{A}_{\lambda 1}\right.$ ") implies that $A^{\lambda}$ is comparable to $-(-\Delta)^{\frac{\alpha}{2}}$, and local shift-boundedness in $\left(\mathrm{A}_{\lambda 1}{ }^{\prime}\right)$ is used to show that functions with finite energy can be approximated by test functions (cf. Theorem 2.6).

(c) By $\left(\mathrm{A}_{\mu}\right)$, the operator $\mathcal{L}^{\mu}$ defined by (1.7) is well-defined on $C^{2}\left(\mathbb{R}^{N}\right) \cap$ $L^{\infty}\left(\mathbb{R}^{N}\right)$, nonpositive and symmetric. The generator of any symmetric pure jump Lévy process is included, like e.g. the fractional Laplacian and symmetric finite difference operators.

(d) If $\lambda(x, \mathrm{~d} z)=\mu(\mathrm{d} z)$, then

$$
\left(\mathrm{A}_{\mu}\right) \quad \Longrightarrow \quad\left(\mathrm{A}_{\lambda 0}\right), \quad\left(\mathrm{A}_{\lambda 1}{ }^{\prime}\right) \text {, and }\left(\mathrm{A}_{\lambda 2}\right) \text {. }
$$

The first two trivially hold, while $\left(\mathrm{A}_{\lambda 2}\right)$ holds by e.g. Lemma 6.4 in [22].

(e) Without loss of generality we can assume $\varphi(0)=0$ (by adding a constant).

2.2. Uniqueness results for energy solutions. In this section we give several uniqueness results for energy (or weak) solutions of (1.1)-(1.2). These results follow from an extension of the Oleinnik argument.

Definition 2.2 (Energy solutions). A function $u \in L_{\mathrm{loc}}^{1}\left(Q_{T}\right)$ is an energy solution of (1.1) -(1.2) if

(i) $\varphi(u) \in L^{2}\left(0, T ; E_{\lambda}\left(\mathbb{R}^{N}\right)\right)$; and

(ii) for all $\psi \in C_{\mathrm{c}}^{\infty}\left(\mathbb{R}^{N} \times[0, T)\right)$,

$$
\int_{0}^{T}\left(\int_{\mathbb{R}^{N}} u \partial_{t} \psi \mathrm{d} x-\mathcal{E}_{\lambda}[\varphi(u), \psi]\right) \mathrm{d} t+\int_{\mathbb{R}^{N}} u_{0}(x) \psi(x, 0) \mathrm{d} x=0 .
$$

Remark 2.3. (a) The integrals in (ii) are well-defined by $\left(\mathrm{A}_{\lambda 0}\right),\left(\mathrm{A}_{\lambda 1}{ }^{\prime}\right),\left(\mathrm{A}_{u_{0}}\right)$, and the regularity of $u$ and $\varphi(u)$. From (ii) it follows that the initial condition $u_{0}$ is assumed in the distributional sense ( $u_{0}$ is a weak initial trace):

$$
\underset{t \rightarrow 0^{+}}{\operatorname{ess} \lim _{\mathbb{R}^{N}}} u(x, t) \psi(x, t) \mathrm{d} x=\int_{\mathbb{R}^{N}} u_{0}(x) \psi(x, 0) \mathrm{d} x \quad \forall \psi \in C_{\mathrm{c}}^{\infty}\left(\mathbb{R}^{N} \times[0, T)\right) .
$$

(b) By the support of the test functions, we could take $L_{\mathrm{loc}}^{2}\left([0, T) ; E_{\lambda}\left(\mathbb{R}^{N}\right)\right)$ in (i).

To state the uniqueness results, we will introduce spaces in which the Oleĭnik argument works. A particular requirement is that test functions are 
dense in these spaces w.r.t. to the weakest convergence that can be used in the proof. This is encoded in the following space:

$$
\begin{aligned}
& X:=\left\{f \in L^{\infty}\left(Q_{T}\right) \cap L^{2}\left(0, T ; E_{\lambda}\left(\mathbb{R}^{N}\right)\right):\right. \\
& \text { there exists }\left\{\psi_{n}\right\}_{n \in \mathbb{N}} \subset C_{\mathrm{c}}^{\infty}\left(\mathbb{R}^{N} \times[0, T)\right) \text { such that } \\
&\left|\psi_{n}-f\right|_{T, E_{\lambda}} \rightarrow 0 \text { as } n \rightarrow \infty \text {, and } \\
&\left.\iint_{Q_{T}} \psi_{n} \phi \mathrm{d} x \mathrm{~d} t \rightarrow \iint_{Q_{T}} f \phi \mathrm{d} x \mathrm{~d} t \text { for all } \phi \in L^{1}\left(Q_{T}\right) \text { as } n \rightarrow \infty\right\} .
\end{aligned}
$$

Below we show that limits can be avoided to get more useful characterizations of such spaces if we (i) go to subspaces, e.g.

$$
X \cap L^{2}\left(Q_{T}\right)=L^{2}\left(Q_{T}\right) \cap L^{\infty}\left(Q_{T}\right) \cap L^{2}\left(0, T ; E_{\lambda}\left(\mathbb{R}^{N}\right)\right) ;
$$

or (ii) restrict the operator by assuming $\left(\mathrm{A}_{\lambda 1}\right.$ ") which implies

$$
X=L^{\infty}\left(Q_{T}\right) \cap L^{2}\left(0, T ; E_{\mu_{\alpha}}\left(\mathbb{R}^{N}\right)\right) .
$$

We refer to Theorem 2.6 below for precise statements.

Our most general uniqueness result applies to energy solutions in the following class of functions:

$$
\mathscr{U} \mathscr{C}:=\left\{u \in L^{1}\left(Q_{T}\right) \cap L^{\infty}\left(Q_{T}\right): \varphi(u) \in X\right\} .
$$

Theorem 2.4 (Uniqueness 1). Assume $\left(\mathrm{A}_{\varphi}\right),\left(\mathrm{A}_{\lambda 0}\right),\left(\mathrm{A}_{\lambda 1}\right)$, and $\left(\mathrm{A}_{u_{0}}\right)$. Then there is at most one energy solution $u$ of (1.1) -(1.2) in $\mathscr{U} \mathscr{C}$.

A proof can be found in Section 3 ,

Remark 2.5. A similar but less general uniqueness result is given by Theorem 1.1 in 247. They assume that $\lambda(x, \mathrm{~d} z)$ is absolutely continuous with a density comparable to the Lévy measure of the $\alpha$-stable process, and hence $A^{\lambda}$ is comparable to $-(-\Delta)^{\frac{\alpha}{2}}$. In this case $\left(\mathrm{A}_{\lambda 1}{ }^{\prime}\right)$ is satisfied in view of the discussion in Section 2.4

Note that in general the uniqueness class $\mathscr{U} \mathscr{C}$ is smaller than the natural existence class

$$
\mathscr{E} \mathscr{C}:=\left\{u \in L^{1}\left(Q_{T}\right) \cap L^{\infty}\left(Q_{T}\right): \varphi(u) \in L^{2}\left(0, T ; E_{\lambda}\left(\mathbb{R}^{N}\right)\right)\right\} .
$$

This is an intrinsic problem with the Oleinnik argument when it is extended to such general settings as we consider here, and it is also observed in 24 . However, the two classes may coincide under additional assumptions, e.g. if $\varphi(u)$ also belongs to $L^{2} \cap L^{\infty}$ or if $A^{\lambda}$ is comparable to $-(-\Delta)^{\frac{\alpha}{2}}$. This is a consequence of the following result. 
Theorem 2.6. Assume $\left(\mathrm{A}_{\lambda 0}\right)$ and $\left(\mathrm{A}_{\lambda 2}\right)$.

(a) If $\left(\mathrm{A}_{\lambda 1}{ }^{\prime}\right)$ holds, then (2.2) holds.

(b) If $\left(\mathrm{A}_{\lambda 1}\right.$ ") holds, then (2.3) holds.

The proofs are given in Appendices $\mathrm{A}$ and $\mathrm{C}$ respectively. See also Section 2.4 for a possible alternative based on recurrence. By Theorem 2.4 and Theorem 2.6, we now have:

Corollary 2.7 (Uniqueness 2). Assume $\left(\mathrm{A}_{\lambda 0}\right),\left(\mathrm{A}_{\lambda 2}\right),\left(\mathrm{A}_{\varphi}\right)$, and $\left(\mathrm{A}_{u_{0}}\right)$ hold.

(a) If $\left(\mathrm{A}_{\lambda 1}\right.$ ') holds, then there is at most one energy solution $u$ of (1.1) -(1.2) such that $u \in \mathscr{E} \mathscr{C}$ and $\varphi(u) \in L^{2}\left(Q_{T}\right)$.

(b) If $\left(\mathrm{A}_{\lambda 1}\right.$ ") holds, then there is at most one energy solution $u$ of (1.1)-(1.2) such that $u \in \mathscr{E} \mathscr{C}$.

Remark 2.8. When the operator $A^{\lambda}$ is comparable to the fractional Laplacian $-(-\Delta)^{\frac{\alpha}{2}}$ for $\alpha \in(0,2)$ (i.e. $\left(\mathrm{A}_{\lambda 1}\right.$ ") holds), the uniqueness and existence classes coincide, and if $N>\alpha$ they satisfy

$$
\mathscr{U} \mathscr{C}=\mathscr{E} \mathscr{C}=\left\{u \in L^{1}\left(Q_{T}\right) \cap L^{\infty}\left(Q_{T}\right): \varphi(u) \in L^{2}\left(0, T ; \dot{H}^{\frac{\alpha}{2}}\left(\mathbb{R}^{N}\right)\right)\right\} .
$$

The latter space is often used in the porous medium setting [43, [26], see also [24]. See Appendix B for rigorous definitions and properties of the homogeneous fractional Sobolev spaces $\dot{H}^{\frac{\alpha}{2}}\left(\mathbb{R}^{N}\right)$ and $L^{2}\left(0, T ; \dot{H}^{\frac{\alpha}{2}}\left(\mathbb{R}^{N}\right)\right)$, some of these we were not able to find in the literature.

Note that if $\left(\mathrm{A}_{\varphi}\right)$ holds and $u \in L^{\infty}\left(Q_{T}\right)$, then $\varphi(u) \in L^{\infty}\left(Q_{T}\right)$. Now let $\beta \in(0,1]$ and assume $\varphi$ is locally $\beta$-Hölder continuous at 0 :

$$
\sup _{|s|<R} \frac{|\varphi(s)-\varphi(0)|}{|s|^{\beta}}<\infty \quad \text { for all } \quad R>0 .
$$

Then, since $\varphi(0)=0$ and $u \in L^{\infty}\left(Q_{T}\right)$,

$$
u \in L^{2 \beta}\left(Q_{T}\right) \quad \Longrightarrow \quad \varphi(u) \in L^{2}\left(Q_{T}\right) .
$$

By interpolation, functions $u \in L^{1} \cap L^{\infty}$ belongs to $L^{2 \beta}$ for $\beta \in\left[\frac{1}{2}, 1\right]$. This leads us to our next result:

Corollary 2.9 (Uniqueness 3$)$. Assume $\left(\mathrm{A}_{\varphi}\right),\left(\mathrm{A}_{\lambda 0}\right),\left(\mathrm{A}_{\lambda 1}{ }^{\prime}\right),\left(\mathrm{A}_{\lambda 2}\right)$, and $\left(\mathrm{A}_{u_{0}}\right)$ hold. If in addition (2.5) holds for some $\beta \in\left[\frac{1}{2}, 1\right]$, then there is at most one energy solution $u$ of (1.1) -(1.2) such that $u \in \mathscr{E} \mathscr{C}$.

Now we specialize to the case $\lambda(x, \mathrm{~d} z)=\mu(\mathrm{d} z)$ and $A^{\lambda}=\mathcal{L}^{\mu}$. Equation (1.1) then becomes equation (1.3). From all the above uniqueness results and Remark 2.1 (d) we obtain the following uniqueness results for (1.3)-(1.4). 
Corollary 2.10 (Uniqueness 4). Assume $\left(\mathrm{A}_{\varphi}\right),\left(\mathrm{A}_{\mu}\right)$, and $\left(\mathrm{A}_{u_{0}}\right)$ hold.

(a) There is at most one energy solution $u$ of (1.3)-(1.4) in $\mathscr{U} \mathscr{C}$.

(b) There is at most one energy solution $u$ of (1.3)-(1.4) such that $u \in \mathscr{E} \mathscr{C}$ and $\varphi(u) \in L^{2}\left(Q_{T}\right)$.

(c) If in addition (2.5) holds for some $\beta \in\left[\frac{1}{2}, 1\right]$, then there is at most one energy solution $u$ of (1.3) -(1.4) such that $u \in \mathscr{E} \mathscr{C}$.

2.3. Equivalence with distributional solutions and consequences. In this section we study the connection between distributional (or very weak) solutions and energy (or weak) solutions. We focus on the simpler case where $A^{\lambda}=\mathcal{L}^{\mu}$, and hence the measure $\lambda(x, \mathrm{~d} z)=\mu(\mathrm{d} z)$ is independent of $x$. In other words, we consider the Cauchy problem (1.3)-(1.4). In general, $A^{\lambda}$ will have an additional drift/convection term compared to $\mathcal{L}^{\mu}$, see Section 2.4. This gives rise to a nonlinear convection term in the equation and the possibility that solutions develop shocks (cf. e.g. 20] and references therein). Whether this happens or not here is not known and another reason to avoid this case now.

We state an equivalence result for the two solution concepts, existence and uniqueness results for distributional solutions with finite energy, and then transport these results from distributional solutions to energy solutions. The uniqueness results of the previous section are transported in the opposite direction, and the different uniqueness results are then compared. We also give quantitative energy and related $L^{p}$-estimates for distributional solutions.

Definition 2.11 (Distributional solutions). A function $u \in L_{\mathrm{loc}}^{1}\left(Q_{T}\right)$ is a distributional solution of (1.3)-(1.4) if

$\iint_{Q_{T}}\left(u \partial_{t} \psi+\varphi(u) \mathcal{L}^{\mu}[\psi]\right) \mathrm{d} x \mathrm{~d} t+\int_{\mathbb{R}^{N}} u_{0}(x) \psi(x, 0) \mathrm{d} x=0 \forall \psi \in C_{\mathrm{c}}^{\infty}\left(\mathbb{R}^{N} \times[0, T)\right)$.

The integral is well-defined under the assumptions $\left(\mathrm{A}_{\varphi}\right),\left(\mathrm{A}_{\mu}\right)$, and $\left(\mathrm{A}_{u_{0}}\right)$ if also $\varphi(u) \in L^{\infty}$ (which is the case when $u \in L^{\infty}$ ). This weaker notion of solutions does not require finite energy, but when the energy is finite, the two notions of solutions will be equivalent.

Theorem 2.12 (Equivalent notions of solutions). Assume $\left(\mathrm{A}_{\varphi}\right),\left(\mathrm{A}_{\mu}\right)$, $u_{0} \in L^{\infty}\left(\mathbb{R}^{N}\right)$, and $u \in L^{\infty}\left(Q_{T}\right)$. Then the following statements are equivalent:

(a) $u$ is an energy solution of (1.3)-(1.4).

(b) $u$ is a distributional solution of (1.3) -(1.4) such that

$$
\varphi(u) \in L^{2}\left(0, T ; E_{\mu}\left(\mathbb{R}^{N}\right)\right) .
$$


We prove this result in Section 4.1. In the setting of this paper, it turns out that there always exists distributional solutions with finite energy.

Theorem 2.13 (Existence 1). Assume $\left(\mathrm{A}_{\varphi}\right),\left(\mathrm{A}_{\mu}\right)$, and $\left(\mathrm{A}_{u_{0}}\right)$. Then there exists a distributional solution $u$ of (1.3)-(1.4) satisfying

(i) $u \in L^{1}\left(Q_{T}\right) \cap L^{\infty}\left(Q_{T}\right) \cap C\left([0, T] ; L_{\text {loc }}^{1}\left(\mathbb{R}^{N}\right)\right)$; and

(ii) $\varphi(u) \in L^{2}\left(0, T ; E_{\mu}\left(\mathbb{R}^{N}\right)\right)$.

This is one of the main results of this paper and will be proven at the end of Section 4.2. For such solutions we have a new uniqueness result by equivalence, Theorem 2.12, and the uniqueness result for energy solutions in Corollary 2.10,

Corollary 2.14 (Uniqueness 5). Assume $\left(\mathrm{A}_{\varphi}\right),\left(\mathrm{A}_{\mu}\right)$, and $\left(\mathrm{A}_{u_{0}}\right)$ hold.

(a) There is at most one distributional solution $u$ of (1.3)-(1.4) in $\mathscr{U} \mathscr{C}$.

(b) There is at most one distributional solution $u$ of (1.3)-(1.4) such that $u \in \mathscr{E} \mathscr{C}$ and $\varphi(u) \in L^{2}\left(Q_{T}\right)$.

(c) If in addition (2.5) holds for some $\beta \in\left[\frac{1}{2}, 1\right]$, then there is at most one distributional solution $u$ of (1.3) -(1.4) such that $u \in \mathscr{E} \mathscr{C}$.

Note that we have uniqueness in a smaller class than we have existence for by Theorem 2.13. This uniqueness result should also be compared to our recent general uniqueness result from $[22$.

Theorem 2.15 (Uniqueness 6, Theorem 2.8 in 22]). Assume $\left(\mathrm{A}_{\varphi}\right),\left(\mathrm{A}_{\mu}\right)$, and $\left(\mathrm{A}_{u_{0}}\right)$. Then there is at most one distributional solution $u$ of (1.3)-(1.4) satisfying

$$
u \in L^{1}\left(Q_{T}\right) \cap L^{\infty}\left(Q_{T}\right) \cap C\left([0, T] ; L_{\mathrm{loc}}^{1}\left(\mathbb{R}^{N}\right)\right) .
$$

In particular, any solution from Theorem 2.13 is unique. This result is more general than Corollary 2.14, but the proof is also more complicated. When Corollary 2.14 applies, a greatly simplified uniqueness argument is available (as we have seen).

In view of the equivalence in Theorem 2.12, we can also transport results in the other direction: from distributional solutions to energy solutions. First we obtain a new existence result as an immediate consequence of Theorem 2.13.

Corollary 2.16 (Existence 2). Assume $\left(\mathrm{A}_{\varphi}\right),\left(\mathrm{A}_{\mu}\right)$, and $\left(\mathrm{A}_{u_{0}}\right)$. Then there exists an energy solution $u$ of (1.3) -(1.4) satisfying

$$
u \in L^{1}\left(Q_{T}\right) \cap L^{\infty}\left(Q_{T}\right) \cap C\left([0, T] ; L_{\mathrm{loc}}^{1}\left(\mathbb{R}^{N}\right)\right) .
$$

In the case of $x$-independent operators, this existence result is much more general than the result given in Theorem 1.1 in [24]. Uniqueness results for energy solutions of (1.3)-(1.4) are given in Corollary 2.10, These results hold 
for a smaller class of functions than the above existence results. However, a (more) general uniqueness result can be obtained from the result for distributional solutions in Theorem 2.15 and equivalence.

Corollary 2.17 (Uniqueness 6). Assume $\left(\mathrm{A}_{\varphi}\right),\left(\mathrm{A}_{\mu}\right)$, and $\left(\mathrm{A}_{u_{0}}\right)$. Then there is at most one energy solution $u$ of (1.3)-(1.4) satisfying

$$
u \in L^{1}\left(Q_{T}\right) \cap L^{\infty}\left(Q_{T}\right) \cap C\left([0, T] ; L_{\mathrm{loc}}^{1}\left(\mathbb{R}^{N}\right)\right) .
$$

The proof is immediate. The solutions of Corollary 2.16 are therefore unique, and this result is stronger than the Olennik type result Corollary 2.10, In view of the well-posedness of both energy and distributional solutions and the equivalence between the two notions of solutions, we now have a full equivalence result under assumptions $\left(\mathrm{A}_{\varphi}\right),\left(\mathrm{A}_{\mu}\right)$, and $\left(\mathrm{A}_{u_{0}}\right)$.

Corollary 2.18 (Equivalent notions of solutions 2). Assume $\left(\mathrm{A}_{\varphi}\right),\left(\mathrm{A}_{\mu}\right)$, $\left(\mathrm{A}_{u_{0}}\right)$, and $u \in L^{1}\left(Q_{T}\right) \cap L^{\infty}\left(Q_{T}\right) \cap C\left([0, T] ; L_{\text {loc }}^{1}\left(\mathbb{R}^{N}\right)\right)$. Then $u$ is an energy solution of (1.3)-(1.4) if and only if it is a distributional solution.

We end this section by new quantitative energy and related $L^{p}$-estimates for the unique distributional solution $u$ provided by Theorems 2.13 and 2.15. This type of estimates are widely used for different local and nonlocal equations of porous medium type, see the discussion in Section 2.4. All proofs are given in Section 4.2. Now, define $\Phi: \mathbb{R} \rightarrow \mathbb{R}$ by $\Phi(w)=\int_{0}^{w} \varphi(\xi) \mathrm{d} \xi$. Then we have:

Theorem 2.19 (Energy inequality). Assume $\left(\mathrm{A}_{\varphi}\right),\left(\mathrm{A}_{\mu}\right)$, and $\left(\mathrm{A}_{u_{0}}\right)$. Then the distributional solution $u$ of (1.3) -(1.4) satisfies

$$
\int_{\mathbb{R}^{N}} \Phi(u(x, \tau)) \mathrm{d} x+|\varphi(u)|_{\tau, E_{\mu}}^{2} \leq \int_{\mathbb{R}^{N}} \Phi\left(u_{0}\right) \mathrm{d} x \quad \text { for } \quad \tau \in(0, T] .
$$

Since $\Phi \geq 0$, we immediately have a quantitative bound on the energy.

Corollary 2.20. Assume $\left(\mathrm{A}_{\varphi}\right),\left(\mathrm{A}_{\mu}\right)$, and $\left(\mathrm{A}_{u_{0}}\right)$. Then the distributional solution $u$ of (1.3) - (1.4) satisfies

$$
|\varphi(u)|_{T, E_{\mu}}^{2} \leq\left\|\varphi\left(u_{0}\right)\right\|_{L^{\infty}\left(\mathbb{R}^{N}\right)}\left\|u_{0}\right\|_{L^{1}\left(\mathbb{R}^{N}\right)}<\infty .
$$

There is also a second type of energy inequality that implies $L^{p}$-bounds.

Theorem 2.21 ( $L^{p}$-bounds). Assume $\left(\mathrm{A}_{\varphi}\right),\left(\mathrm{A}_{\mu}\right)$, and $\left(\mathrm{A}_{u_{0}}\right)$. Then the distributional solution $u$ of (1.3) (1.4) satisfies, for $0<\tau \leq T$,

$$
\int_{\mathbb{R}^{N}}|u(x, \tau)|^{p} \mathrm{~d} x \leq \int_{\mathbb{R}^{N}}\left|u_{0}(x)\right|^{p} \mathrm{~d} x \quad \text { for all } \quad p \in[1, \infty),
$$

and in the case $p=\infty$,

$$
\|u(\cdot, \tau)\|_{L^{\infty}\left(\mathbb{R}^{N}\right)} \leq\left\|u_{0}\right\|_{L^{\infty}\left(\mathbb{R}^{N}\right)} .
$$




\subsection{Remarks.}

Locally shift-bounded kernels. Let $\mu(\mathrm{d} z)$ be a nonnegative locally finite Borel measure on $\mathbb{R}^{N} \backslash\{0\}$ and $j(x, z)$ a measurable function satisfying

$$
0<m \leq j(x, z) \leq M<\infty .
$$

Then the kernel

$$
\lambda(x, \mathrm{~d} z)=j(x, z) \mu(\mathrm{d} z)
$$

is not only locally, but also globally, shift-bounded in the sense that for all $x, h \in \mathbb{R}^{N}$ and Borel $B \subset \mathbb{R}^{N} \backslash\{0\}$,

$$
\frac{\lambda(x+h, B)}{\lambda(x, B)} \leq \frac{M}{m} .
$$

Examples of $\mu$ are Lévy measures of Lévy processes, e.g. $\mu(\mathrm{d} z)=\frac{c_{N, \alpha}}{|z|^{N+\alpha}} \mathrm{d} z$ for the $\alpha$-stable process $(\alpha \in(0,2))$ with the fractional Laplacian as generator. The latter case corresponds exactly to assumption $\left(\mathrm{A}_{\lambda 1} "\right)$.

Recurrence and alternative characterization of $\boldsymbol{X}$. In Theorem 2.6 (a) approximation by test functions is obtained by an additional assumption on the function class. Alternatively, as in part (b), we can keep the original function class, but restrict the bilinear form $\mathcal{E}_{\lambda}$ (and hence the generator $A^{\lambda}$ ). In the elliptic setting such results are given in Theorem 3.2 in [36] under the assumptions that $\left(\mathrm{A}_{\lambda 0}\right),\left(\mathrm{A}_{\lambda 1}{ }^{\prime}\right)$ and $\left(\mathrm{A}_{\lambda 2}\right)$ hold and the closure of $\left(\mathcal{E}_{\lambda}, C_{\mathrm{c}}^{\infty}\left(\mathbb{R}^{N}\right)\right)$ is recurrent. A condition ensuring recurrence for symmetric Lévy processes is given in Section 37 in [34]. E.g. the fractional Laplacian $-(-\Delta)^{\frac{\alpha}{2}}$ for $\alpha \in(0,2)$ is recurrent if $N \leq \alpha$-which is a rather restrictive assumption! Similar results are true in our parabolic setting. Assuming recurrence, or rather, assuming existence of the sequence of cut-off functions mentioned in Lemma 3.1 in [36, we get

$$
X=L^{\infty}\left(Q_{T}\right) \cap L^{2}\left(0, T ; E_{\lambda}\left(\mathbb{R}^{N}\right)\right) .
$$

The proof is an easy modification of the proof of Theorem 3.2 in [36] if we assume $\left(\mathrm{A}_{\lambda 0}\right),\left(\mathrm{A}_{\lambda 1}{ }^{\prime}\right)$, and $\left(\mathrm{A}_{\lambda 2}\right)$ hold (as in Theorem [2.6 (a)) and, in addition, $\iint|x-y|^{2} \Lambda(\mathrm{d} x, \mathrm{~d} y)<\infty$. Note that the latter condition implies $\int_{0}^{T} \mathcal{E}_{\lambda}\left[\phi_{n}, \phi_{n}\right] \mathrm{d} t \rightarrow 0$ for any $\phi_{n} \in C_{\mathrm{c}}^{1}\left(Q_{T}\right)$ such that $\phi_{n} \rightarrow 1$ a.e. and $\left\|D \phi_{n}\right\|_{L^{\infty}} \rightarrow$ 0 . However, this extra condition excludes all Lévy processes and all $x$-independent generators.

Integral representations of the operators $A^{\lambda}$. In general the operator $A^{\lambda}$ is abstractly defined from $\mathcal{E}_{\lambda}$ by formula (1.6). However explicit integral representation formulas exist under additional assumptions on the kernel $\lambda(x, \mathrm{~d} z)$ (cf. (2.1)). We follow [35] and assume $\left(\mathrm{A}_{\lambda 0}\right)$ and $\left(\mathrm{A}_{\lambda 1}\right)$ hold and

$$
\lambda(x, \mathrm{~d} z)=\tilde{\lambda}(x, x+\mathrm{d} z)=j(x, x+z) \mathrm{d} z,
$$


where $j \geq 0$ is a symmetric measurable function on $\mathbb{R}^{N} \times \mathbb{R}^{N} \backslash\{x\}$ such that

$$
\int_{|z| \leq 1}|z||j(x, x+z)-j(x, x-z)| \mathrm{d} z<\infty .
$$

Symmetric here means that $j(x, y)=j(y, x)$. Note that now $\Lambda(\mathrm{d} x, \mathrm{~d} y)=$ $j(x, y) \mathrm{d} x \mathrm{~d} y$ in (1.5). By Theorem 2.2 in [35], it then follows that

$$
\begin{aligned}
A^{\lambda}[\phi](x):= & \int_{|z|>0}\left(\phi(x+z)-\phi(x)-z \cdot D \phi(x) \mathbf{1}_{|z| \leq 1}\right) j(x, x+z) \mathrm{d} z \\
& +\frac{1}{2} \int_{|z|>0} z \mathbf{1}_{|z| \leq 1}(j(x, x+z)-j(x, x-z)) \mathrm{d} z \cdot D \phi(x)
\end{aligned}
$$

for $\phi \in C_{\mathrm{c}}^{2}\left(\mathbb{R}^{N}\right)$. Compare with (1.7) and note that the second integral is like a drift term that vanishes if $j(x, x+z)=j(x, x-z)$. Under slightly stronger assumptions, this $A^{\lambda}$ coincides on $C_{\mathrm{c}}^{2}\left(\mathbb{R}^{N}\right)$ with the generator of the closure of $\left(\mathcal{E}_{\lambda}, C_{\mathrm{c}}^{\infty}\left(\mathbb{R}^{N}\right)\right)$ in $L^{2}\left(\mathbb{R}^{N}\right)$ - see Proposition 2.5 in 35 .

Let us simplify and assume that

$$
j(x, y)=j_{1}(x, y) \mu(x-y)
$$

for $j_{1}$ symmetric, $j_{1}(x, x+z)=j_{1}(x, x-z)$, and $\mu$ even. This $j$ is symmetric and $j(x, x+z)=j(x, x-z)$. Taking $j_{1}(x, y)=a(x)+a(y)$ and $\mu(z)=\frac{c_{N, \alpha}}{|z|^{N+\alpha}}$, the Lévy density of the fractional Laplacian, we get an $x$-depending fractional Laplace like operator:

$$
\begin{aligned}
A_{1}^{\lambda}[\phi](x)= & -a(x)(-\Delta)^{\frac{\alpha}{2}} \phi(x) \\
& +\int_{|z|>0}\left(\phi(x+z)-\phi(x)-z \cdot D \phi(x) \mathbf{1}_{|z| \leq 1}\right) a(x+z) \frac{c_{N, \alpha}}{|z|^{N+\alpha}} \mathrm{d} z .
\end{aligned}
$$

From this example we also learn that our class of operators does not include the simplest and most natural $x$-depending fractional Laplace operator,

$$
-a(x)(-\Delta)^{\frac{\alpha}{2}} \phi(x),
$$

since it only satisfies the symmetry assumption on $j\left(\right.$ or $\left.\left(\mathrm{A}_{\lambda 2}\right)\right)$ if $a$ is constant!

On $\boldsymbol{L}^{p}$-estimates. If $\varphi(u)=u|u|^{m-1}$ and $\mathcal{L}^{\mu}=-(-\Delta)^{\frac{\sigma}{2}}$, then by [26] the estimate corresponding to Theorem 2.21 takes the form

$$
\int_{\mathbb{R}^{N}}|u(x, \tau)|^{p} \mathrm{~d} x+\left.\left.\int_{0}^{\tau} \int_{\mathbb{R}^{N}}\left|(-\Delta)^{\frac{\sigma}{4}}\right| u\right|^{\frac{p+m-1}{2}}\right|^{2} \mathrm{~d} x \mathrm{~d} t \leq \int_{\mathbb{R}^{N}}\left|u_{0}(x)\right|^{p} \mathrm{~d} x .
$$

Note the additional energy term. A closer look at our proof, see Corollary 4.12 and the proof of Theorem 2.21, reveals that we could also have an $L^{p}$-estimate 
with some energy. However, this energy is only a limit and hard to characterize under our weak assumptions.

Such $L^{p}$ type decay estimates are an essential tool for nonlinear diffusion equations of porous medium type. They imply that $|u|^{\frac{p+m-1}{2}}$ belongs to some Sobolev space. This estimate and the Nash-Gagliardo-Nirenberg inequality can be used in a Moser iteration argument to obtain an $L^{1}-L^{\infty}$ smoothing effect and then existence of energy solutions with initial data merely in $L^{1}$ [42, 43, 26, 27, 24]. The other main application of the $L^{p}$-energy estimates is as key steps in Sobolev or Simon type compactness arguments. Such arguments are used in 42, 10, 9, 38, 39, 40 to prove existence of energy solutions through the resolution of a sequence of smooth approximate problems and passing to the limit in view of compactness.

\section{Proof of uniqueness for energy solutions}

In this section we prove Theorem 2.4. We start by some preliminary results.

Lemma 3.1 (Cauchy-Schwartz). Assume $\left(\mathrm{A}_{\lambda 1}\right)$. If $f, g \in L^{2}\left(0, T ; E_{\lambda}\left(\mathbb{R}^{N}\right)\right)$, then

$$
\left|\int_{0}^{T} \mathcal{E}_{\lambda}[f(\cdot, t), g(\cdot, t)] \mathrm{d} t\right| \leq|f|_{T, E_{\lambda}}|g|_{T, E_{\lambda}} .
$$

The proof is as for the classical Cauchy-Schwartz and we omit it.

Lemma 3.2. Assume $\left(\mathrm{A}_{\lambda 1}\right)$. If $f \in L^{2}\left(0, T ; E_{\lambda}\left(\mathbb{R}^{N}\right)\right)$ and $g(x, t)=\int_{t}^{T} f(x, s) \mathrm{d} s$, then $|g|_{T, E_{\lambda}}^{2} \leq \frac{T^{2}}{2}|f|_{T, E_{\lambda}}^{2}$.

Proof. By Jensen's inequality and Tonelli's lemma,

$$
\begin{aligned}
|g|_{T, E_{\lambda}}^{2} & \leq \int_{0}^{T} \frac{1}{2} \iint(T-t) \int_{t}^{T}|f(x+z, s)-f(x, s)|^{2} \mathrm{~d} s \lambda(x, \mathrm{~d} z) \mathrm{d} x \mathrm{~d} t \\
& =\int_{0}^{T}(T-t)\left(\int_{t}^{T}|f(\cdot, s)|_{E_{\lambda}}^{2} \mathrm{~d} s\right) \mathrm{d} t,
\end{aligned}
$$

and the result follows.

Since an energy solution has some regularity, the weak formulation of the equation will hold also with less regular test functions. We will now formulate such a type of result in the relevant setting for the Oleunik argument. 
Lemma 3.3. Let $u$ be an energy solution of (1.1)-(1.2). If $u \in L^{1}\left(Q_{T}\right)$, $u_{0} \in L^{1}\left(\mathbb{R}^{N}\right)$, and $\varphi(u) \in L^{2}\left(0, T ; E_{\lambda}\left(\mathbb{R}^{N}\right)\right)$, then for any $\phi \in X$,

$$
\begin{aligned}
& \int_{0}^{T}\left(-\int_{\mathbb{R}^{N}} u \phi\right.\left.\mathrm{d} x-\mathcal{E}_{\lambda}\left[\varphi(u), \int_{t}^{T} \phi(\cdot, s) \mathrm{d} s\right]\right) \mathrm{d} t \\
&+\int_{\mathbb{R}^{N}} u_{0}(x)\left(\int_{0}^{T} \phi(x, s) \mathrm{d} s\right) \mathrm{d} x=0 .
\end{aligned}
$$

In other words, we may take $\psi(x, t)=\int_{t}^{T} \phi(x, s) \mathrm{d} s$ in Definition 2.2 for $\phi \in X$. Note that the integrals are well-defined: see Lemma 3.2. From the proof below it follows that the choice of space $X$ is (close to) optimal.

Proof. From the definition of $X$ there is $C_{\mathrm{c}}^{\infty}\left(\mathbb{R}^{N} \times[0, T)\right) \ni \phi_{n} \rightarrow \phi \in X$ for the convergence in $X$ as $n \rightarrow \infty$. Let

$$
\psi(x, t):=\int_{t}^{T} \phi(x, s) \mathrm{d} s \quad \text { and } \quad \psi_{n}(x, t):=\int_{t}^{T} \phi_{n}(x, s) \mathrm{d} s .
$$

Observe that $\psi_{n} \in C_{\mathrm{c}}^{\infty}\left(\mathbb{R}^{N} \times[0, T)\right)$ since $\phi_{n}$ is. By Cauchy-Schwartz' inequality, Lemma 3.2, and the convergence in $X$, we see that

$$
\int_{0}^{T} \mathcal{E}_{\lambda}\left[\varphi(u),\left(\psi_{n}-\psi\right)\right] \mathrm{d} t \leq|\varphi(u)|_{T, E_{\lambda}} \frac{T^{2}}{2}\left|\phi_{n}-\phi\right|_{T, E_{\lambda}} \rightarrow 0 \quad \text { as } \quad n \rightarrow \infty .
$$

Since $u \in L^{1}\left(Q_{T}\right)$ and $\phi_{n}$ converges in $X$, we also have

$$
\iint_{Q_{T}} u\left(\partial_{t} \psi_{n}-\partial_{t} \psi\right) \mathrm{d} x \mathrm{~d} t=-\iint_{Q_{T}} u\left(\phi_{n}-\phi\right) \mathrm{d} x \mathrm{~d} t \rightarrow 0 \quad \text { as } \quad n \rightarrow \infty .
$$

In a similar way, $\int_{\mathbb{R}^{N}} u_{0}(x)\left(\psi_{n}-\psi\right)(x, 0) \mathrm{d} x \rightarrow 0$. The result now follows from taking $\psi=\psi_{n}$ in the definition of energy solutions (Definition 2.2), and using the above estimates to pass to the limit.

Remark 3.4. A closer inspection of the proof reveals that strong $|\cdot|_{T, E_{\lambda}}$ convergence cannot be replaced by the corresponding weak convergence. The reason is that the weak convergence property for the test functions $\phi_{n}$ is lost when they are integrated in time to yield the $\psi_{n}$ 's.

Note that for the proof of Lemma 3.3. the definition of $X$ is essential in the sense that we take those functions which can be approximated by $C_{\mathrm{c}}^{\infty}$ functions. This lemma is crucial in the Olennik argument below because we want to take

$$
\psi(x, t)=\int_{t}^{T}(\varphi(u)-\varphi(v))(x, s) \mathrm{d} s
$$

as a test function. By Lemma 3.3, we need that $\varphi(u), \varphi(v) \in X$ for this to be possible, and this explains this strange assumption and space. 
Proof of Theorem 2.4 (Uniqueness 1). Assume there are two different energy solutions $u$ and $v$ of (1.1) with the same initial data (1.2). Let $U=u-v$ and $\Phi=\varphi(u)-\varphi(v)$, and note that the proof is complete if we can show that $U=0$ a.e. in $Q_{T}$.

To show that, we subtract the energy formulation of the equations for $u$ and $v$ (Definition 2.2). Since the initial data are the same, this leads to

$$
\int_{0}^{T}\left(\int_{\mathbb{R}^{N}} U \partial_{t} \psi \mathrm{d} x-\mathcal{E}_{\lambda}[\Phi, \psi]\right) \mathrm{d} t=0 \quad \text { for all } \quad \psi \in C_{\mathrm{c}}^{\infty}\left(\mathbb{R}^{N} \times[0, T)\right) .
$$

Now we adapt the classical argument of Oleinnik et al. 32 and seek to take

$$
\zeta(x, t)= \begin{cases}\int_{t}^{T} \Phi(x, s) \mathrm{d} s & 0 \leq t<T \\ 0 & t \geq T\end{cases}
$$

as a test function. Since $\Phi \in X$ (by the definition of $\mathscr{U} \mathscr{C}$ ), this can be done by Lemma 3.3, and hence

$$
\int_{0}^{T}\left(-\int_{\mathbb{R}^{N}} U \Phi \mathrm{d} x-\mathcal{E}_{\lambda}[\Phi, \zeta]\right) \mathrm{d} t=0 .
$$

Since $\int_{0}^{T}\left|\mathcal{E}_{\lambda}[\Phi, \zeta]\right| \mathrm{d} t<\infty$ by Lemma 3.2, we have by Fubini's theorem

$$
\begin{aligned}
& \int_{0}^{T} \mathcal{E}_{\lambda}[\Phi, \zeta] \mathrm{d} t \\
& =\frac{1}{2} \int_{0}^{T} \int_{\mathbb{R}^{N}} \int_{|z|>0}(\Phi(x+z, t)-\Phi(x, t))(\zeta(x+z)-\zeta(x)) \lambda(x, \mathrm{~d} z) \mathrm{d} x \mathrm{~d} t \\
& =\frac{1}{2} \int_{\mathbb{R}^{N}} \int_{|z|>0} \int_{0}^{T}(\Phi(x+z, t)-\Phi(x, t)) \times \\
& \quad \times \int_{t}^{T}(\Phi(x+z, s)-\Phi(x, s)) \mathrm{d} s \mathrm{~d} t \lambda(x, \mathrm{~d} z) \mathrm{d} x .
\end{aligned}
$$

Then by the identity for $F \in L^{1}((0, T))$,

$$
\int_{0}^{T} F(t)\left(\int_{t}^{T} F(s) \mathrm{d} s\right) \mathrm{d} t=\int_{0}^{T} \int_{t}^{T} F(t) F(s) \mathrm{d} s \mathrm{~d} t=\frac{1}{2}\left(\int_{0}^{T} F(t) \mathrm{d} t\right)^{2}
$$

(follows easily since $\int_{0}^{T} \int_{t}^{T} \ldots \mathrm{d} s \mathrm{~d} t=\int_{0}^{T} \int_{0}^{s} \ldots \mathrm{d} t \mathrm{~d} s$ ),

$$
\int_{0}^{T} \mathcal{E}_{\lambda}[\Phi, \zeta] \mathrm{d} t=\frac{1}{4} \int_{\mathbb{R}^{N}} \int_{|z|>0}\left(\int_{0}^{T}(\Phi(x+z, t)-\Phi(x, t)) \mathrm{d} t\right)^{2} \lambda(x, \mathrm{~d} z) \mathrm{d} x \geq 0 .
$$


Returning to (3.2), we then find that

$$
\int_{0}^{T} \int_{\mathbb{R}^{N}} U \Phi \mathrm{d} x \mathrm{~d} t \leq 0
$$

Since $\varphi$ is nondecreasing by $\left(\mathrm{A}_{\varphi}\right), U \Phi \geq 0$ a.e., and it then follows that $U \Phi=0$ a.e. in $Q_{T}$. This means that at a.e. point, either $U=0$ or $\Phi=0$, and hence since $U=0$ implies $\Phi=0$ by definition,

$$
\Phi=0 \quad \text { a.e. in } Q_{T} .
$$

Then by equation (3.1),

$$
\int_{0}^{T} \int_{\mathbb{R}^{N}} U \partial_{t} \psi \mathrm{d} x \mathrm{~d} t=0 \quad \text { for all } \quad \psi \in C_{\mathrm{c}}^{\infty}\left(\mathbb{R}^{N} \times[0, T)\right) .
$$

Since $\psi(x, t):=\int_{t}^{T} \phi(x, s) \mathrm{d} s \in C_{\mathrm{c}}^{\infty}\left(\mathbb{R}^{N} \times[0, T)\right)$ for arbitrary $\phi \in C_{\mathrm{c}}^{\infty}\left(Q_{T}\right)$,

$$
-\int_{0}^{T} \int_{\mathbb{R}^{N}} U \phi \mathrm{d} x \mathrm{~d} t=0 \quad \text { for all } \quad \phi \in C_{\mathrm{c}}^{\infty}\left(Q_{T}\right),
$$

and hence $U=0$ a.e. in $Q_{T}$ by du Bois-Reymond's lemma.

\section{Distributional solutions with finite energy}

Our main focus in this section is to prove Theorems 2.12, 2.13, 2.19, and 2.21, First, we prove the equivalence of notions of solutions. Second, we consider an approximate problem of (1.3)-(1.4). The energy and $L^{p}$-estimates are then shown to hold for the solution of that problem. A compactness result will give us convergence of solutions of the approximate problem, and we thus obtain existence of some limit solution of the full problem satisfying the same estimates.

We recall that (i) $\mathcal{L}^{\mu}[\psi]$ is well-defined for $\psi \in C^{2}\left(\mathbb{R}^{N}\right) \cap L^{\infty}\left(\mathbb{R}^{N}\right)$; (ii) $\mathcal{L}^{\mu}[\psi]$ is bounded in $L^{1} / L^{\infty}$ for $\psi \in W^{2,1} / W^{2, \infty}$; and (iii) $\mathcal{L}^{\mu}$ is symmetric for e.g. functions in $W^{2,1} / W^{2, \infty}$ (see Lemma 3.5 in [22]). Note also that for $\mu$ replaced by $\mu_{r}:=\mu \mathbf{1}_{|z|>r}$, (i)-(iii) holds when we only assume that $\psi$ is in $L^{\infty}, L^{1} / L^{\infty}$, and $L^{1} / L^{\infty}$ (see Remark 3.6 (b) in [22]).

4.1. Equivalent notions of solutions. We establish the relation between the ( $x$-independent) bilinear form and our Lévy operator, as a consequence, we get equivalence of energy and distributional solutions under certain conditions. 
Proposition 4.1. Assume $\left(\mathrm{A}_{\mu}\right)$. For any $\psi \in C_{\mathrm{c}}^{\infty}\left(\mathbb{R}^{N}\right)$, and $v \in L^{\infty}\left(\mathbb{R}^{N}\right) \cap$ $E_{\mu}\left(\mathbb{R}^{N}\right)$, we have

$$
\begin{aligned}
& \int_{\mathbb{R}^{N}} v(x) \mathcal{L}^{\mu}[\psi](x) \mathrm{d} x \\
& =-\frac{1}{2} \int_{\mathbb{R}^{N}} \int_{|z|>0}(v(x+z)-v(x))(\psi(x+z)-\psi(x)) \mu(\mathrm{d} z) \mathrm{d} x=-\mathcal{E}_{\mu}[v, \psi] .
\end{aligned}
$$

Remark 4.2. The result holds as long as both sides make sense.

Lemma 4.3. Assume that $\nu \geq 0$ is an even Radon measure with $\nu\left(\mathbb{R}^{N}\right)<\infty$, and $1 \leq p, q \leq \infty$ with $\frac{1}{p}+\frac{1}{q}=1$. For any $f \in L^{p}\left(\mathbb{R}^{N}\right)$ and $g \in L^{q}\left(\mathbb{R}^{N}\right)$, we have

$$
\int_{\mathbb{R}^{N}} g(x) \mathcal{L}^{\nu}[f](x) \mathrm{d} x=-\mathcal{E}_{\nu}[f, g] .
$$

This proof is postponed to Appendix D,

Proof of Proposition 4.1. Replace $\nu$ by $\mu_{r}=\mu \mathbf{1}_{|z|>r}$ in Lemma 4.3, and let $g=v$ and $f=\psi$. Then the result follows by Lebesgue's dominated convergence theorem as $r \rightarrow 0^{+}$since $\mathbf{1}_{|z|>r} \leq 1$.

Proof of Theorem 2.12 (Equivalent notions of solutions). (a) $\Longrightarrow(b)$ In Definition 2.2, we have that $|\varphi(u)|_{T, E_{\mu}}<\infty$, and then we can use Proposition 4.1 to obtain (note that $\varphi(u) \in L^{\infty}\left(\mathbb{R}^{N}\right)$ )

$\int_{0}^{T} \int_{\mathbb{R}^{N}} u \partial_{t} \psi+\varphi(u) \mathcal{L}^{\mu}[\psi] \mathrm{d} x \mathrm{~d} t+\int_{\mathbb{R}^{N}} u_{0}(x) \psi(x, 0) \mathrm{d} x=0 \forall \psi \in C_{\mathrm{c}}^{\infty}\left(\mathbb{R}^{N} \times[0, T)\right)$.

$(b) \Longrightarrow(a)$ We write Definition 2.11 in the following way

$$
\begin{aligned}
\int_{0}^{T}\left(\int_{\mathbb{R}^{N}} u \partial_{t} \psi \mathrm{d} x+\int_{\mathbb{R}^{N}} \varphi(u) \mathcal{L}^{\mu}[\psi] \mathrm{d} x\right) \mathrm{d} t & \\
& +\int_{\mathbb{R}^{N}} u_{0}(x) \psi(x, 0) \mathrm{d} x=0 \quad \forall \psi \in C_{\mathrm{c}}^{\infty}\left(\mathbb{R}^{N} \times[0, T)\right) .
\end{aligned}
$$

By the assumptions, $|\varphi(u)|_{T, E_{\mu}}<\infty$, and hence, we can use Proposition 4.1 in the other direction to get energy solutions.

4.2. The approximate problem of (1.3)-(1.4). By using a priori and existence results for a simplified version of (1.3)-(1.4), we can take the limit of a sequence of solutions of such problems, and then conclude that some limit solution of the full problem exists and enjoys the energy and $L^{p}$-estimates.

Let $\omega_{n}$ be a family of mollifiers defined by

$$
\omega_{n}(\sigma):=n^{N} \omega(n \sigma)
$$


for fixed $0 \leq \omega \in C_{\mathrm{c}}^{\infty}\left(\mathbb{R}^{N}\right)$ with $\operatorname{supp} \omega \subseteq \bar{B}(0,1), \omega(\sigma)=\omega(-\sigma), \int \omega=1$, and define

$$
\varphi_{n}(x):=\varphi * \omega_{n}(x)-\varphi * \omega_{n}(0) \text { where } \omega_{n} \text { is given by (4.1) with } N=1 \text {. }
$$

Now, consider the following approximation of (1.3)-(1.4) where the measure $\mu$ is replaced by $\mu_{r}=\mu \mathbf{1}_{|z|>r}$ and the nonlinear diffusion flux $\varphi$ is replaced by $\varphi_{n}$ :

$$
\begin{aligned}
\partial_{t} u_{r, n}-\mathcal{L}^{\mu_{r}}\left[\varphi_{n}\left(u_{r, n}\right)\right] & =0 & & \text { in } \quad Q_{T}, \\
u_{r, n}(x, 0) & =u_{0}(x) & & \text { on } \mathbb{R}^{N},
\end{aligned}
$$

with

$$
\mathcal{L}^{\mu_{r}}[\psi](x)=\int_{|z|>0}(\psi(x+z)-\psi(x)) \mu_{r}(\mathrm{~d} z) .
$$

Note that $\varphi_{n} \in C^{\infty}(\mathbb{R})$ (and hence, locally Lipschitz), $\varphi_{n}(0)=0$, and $\varphi_{n} \rightarrow \varphi$ locally uniformly on $\mathbb{R}$ by $\left(\mathrm{A}_{\varphi}\right)$, the properties of mollifiers, and Remark 2.1 (f). Furthermore, recall that for any $r>0$, the operator $\mathcal{L}^{\mu_{r}}[\psi]$ is well-defined for merely bounded $\psi$.

Remark 4.4. Since (4.3)-(4.4) is just a special case of (1.3)-(1.4), existence, uniqueness, (uniform) $L^{1}$-, $L^{\infty}$-bounds, and time regularity holds for (4.3)(4.4) by Theorem 2.10 in [22] or by [23] through limit procedures and compactness results for entropy or numerical solutions.

Theorem 4.5 (Existence and uniqueness, Theorem 2.8 in [22]). Assume $\left(\mathrm{A}_{\varphi}\right)$, $\left(\mathrm{A}_{\mu}\right)$, and $\left(\mathrm{A}_{u_{0}}\right)$. Then there exists a unique distributional solution $u_{r, n}$ of (4.3) -(4.4) satisfying

$$
u_{r, n} \in L^{1}\left(Q_{T}\right) \cap L^{\infty}\left(Q_{T}\right) \cap C\left([0, T] ; L_{\mathrm{loc}}^{1}\left(\mathbb{R}^{N}\right)\right) .
$$

Now we first prove that (4.3) holds a.e., and then we deduce energy and clean $L^{p}$-estimates (the latter by a Stroock-Varopoulos type result) from the rather general inequality in Proposition 4.7 below.

Lemma 4.6. Assume $\left(\mathrm{A}_{\varphi}\right),\left(\mathrm{A}_{\mu}\right)$, and $\left(\mathrm{A}_{u_{0}}\right)$. Then the distributional solution $u_{r, n}$ of (4.3) - (4.4) with initial data $u_{0}$ satisfies

$$
\partial_{t} u_{r, n} \in L^{1}\left(Q_{T}\right) \cap L^{\infty}\left(Q_{T}\right) \quad \text { and } \quad \partial_{t} u_{r, n}=\mathcal{L}^{\mu_{r}}\left[\varphi_{n}\left(u_{r, n}\right)\right] \text { a.e. in } Q_{T} .
$$

Proof. By the definition of distributional solutions for (4.3)-(4.4) and the symmetry of $\mathcal{L}^{\mu_{r}}$,

$-\iint_{Q_{T}} u_{r, n} \partial_{t} \psi \mathrm{d} x \mathrm{~d} t=\iint_{Q_{T}} \varphi_{n}\left(u_{r, n}\right) \mathcal{L}^{\mu_{r}}[\psi] \mathrm{d} x \mathrm{~d} t=\iint_{Q_{T}} \mathcal{L}^{\mu_{r}}\left[\varphi_{n}\left(u_{r, n}\right)\right] \psi \mathrm{d} x \mathrm{~d} t$. 
Hence, $\mathcal{L}^{\mu_{r}}\left[\varphi_{n}\left(u_{r, n}\right)\right]$ is the weak time derivative of $u_{r, n}$. Since $\varphi_{n} \in W^{1, \infty}(\mathbb{R})$, $\varphi_{n}\left(u_{r, n}\right) \in L^{1} \cap L^{\infty}$ and hence, we get that $g:=\mathcal{L}^{\mu_{r}}\left[\varphi_{n}\left(u_{r, n}\right)\right] \in L^{1}\left(Q_{T}\right) \cap$ $L^{\infty}\left(Q_{T}\right)$.

Assume also $u_{r, n} \in C^{1}$. Then $\partial_{t} u_{r, n}=g$ and we can use the Fundamental theorem of calculus to see that

$$
\left\|\frac{u_{r, n}(\cdot, \cdot+h)-u_{r, n}}{h}-g\right\|_{L^{1}\left(Q_{T}\right)} \leq \int_{0}^{1}\|g(\cdot, \cdot+s h)-g\|_{L^{1}\left(Q_{T}\right)} \mathrm{d} s .
$$

By an approximation argument in $L^{1}$, this inequality holds also without the $C^{1}$ assumption. Taking the limit as $h \rightarrow 0^{+}$(the right-hand side goes to zero by Lebesgue's dominated convergence theorem since translations in $L^{1}$ are continuous), we obtain that

$$
\lim _{h \rightarrow 0^{+}} \frac{u_{r, n}(x, t+h)-u_{r, n}(x, t)}{h}=g(x, t) \quad \text { in } L^{1}\left(Q_{T}\right),
$$

and hence, $\partial_{t} u_{r, n}$ exists and equals $g$ a.e. in $Q_{T}$.

To prove the next result, we need to define cut-off functions: Consider $\mathcal{X} \in C_{\mathrm{c}}^{\infty}\left(\mathbb{R}^{N}\right)$ such that $\mathcal{X} \geq 0, \mathcal{X}=1$ when $|x| \leq 1$, and $\mathcal{X}=0$ when $|x|>2$, and define

$$
\mathcal{X}_{R}(\cdot):=\mathcal{X}\left(\frac{\dot{R}}{R}\right) \in C_{\mathrm{c}}^{\infty}\left(\mathbb{R}^{N}\right) \quad \text { for } \quad R>0 .
$$

Proposition 4.7. Assume $\left(\mathrm{A}_{\varphi}\right),\left(\mathrm{A}_{\mu}\right),\left(\mathrm{A}_{u_{0}}\right)$, and $0<\tau \leq T$. Let $\Psi \in$ $W_{\mathrm{loc}}^{1, \infty}(\mathbb{R})$ with $\Psi(0)=0$. Then the distributional solution $u_{r, n}$ of (4.3)-(4.4) satisfies

$$
\begin{aligned}
& \int_{\mathbb{R}^{N}} \Psi\left(u_{r, n}(x, \tau)\right) \mathrm{d} x-\int_{0}^{\tau} \int_{\mathbb{R}^{N}} \Psi^{\prime}\left(u_{r, n}(x, t)\right) \mathcal{L}^{\mu_{r}}\left[\varphi_{n}\left(u_{r, n}(x, t)\right)\right] \mathrm{d} x \mathrm{~d} t \\
& \quad=\int_{\mathbb{R}^{N}} \Psi\left(u_{0}(x)\right) \mathrm{d} x .
\end{aligned}
$$

Remark 4.8. On page 1256 in [26], a similar result as the above is obtained for $\Psi(u)$ nonnegative, nondecreasing and convex.

Proof. Observe that we may assume $\Psi \in W^{1, \infty}(\mathbb{R})$ since $\Psi \in W_{\text {loc }}^{1, \infty}(\mathbb{R})$ and $u_{r, n}, u_{0} \in L^{\infty}$. By Lemma 4.6, $\partial_{t} u_{r, n}=\mathcal{L}^{\mu_{r}}\left[\varphi_{n}\left(u_{r, n}\right)\right]$ a.e. in $Q_{T}$. Multiply this a.e.-equation by $\Psi^{\prime}\left(u_{r, n}(x, t)\right) \mathcal{X}_{R}(x)$ (where $\mathcal{X}_{R}$ is defined in (4.5)) and integrate (in $x$ ) over $\mathbb{R}^{N}$ to get

$$
\int_{\mathbb{R}^{N}} \partial_{t} u_{r, n} \Psi^{\prime}\left(u_{r, n}\right) \mathcal{X}_{R} \mathrm{~d} x=\int_{\mathbb{R}^{N}} \mathcal{L}^{\mu_{r}}\left[\varphi_{n}\left(u_{r, n}\right)\right] \Psi^{\prime}\left(u_{r, n}\right) \mathcal{X}_{R} \mathrm{~d} x .
$$

By Lemma 4.6] and the Sobolev chain rule given by Theorem 2.1.11 in [44], the left-hand side equals $\int_{\mathbb{R}^{N}} \partial_{t} \Psi\left(u_{r, n}\right) \mathcal{X}_{R} \mathrm{~d} x$. Note that the function $\mathcal{X}_{R}$ converges 
pointwise to 1 , is bounded by 1 , and is integrable. Hence we can move the time derivative outside the integral on the left-hand side by Lebesgue's dominated convergence theorem and Lemma 4.6 since $\left|\partial_{t} \Psi\left(u_{r, n}\right) \mathcal{X}_{R}\right| \in L^{1}\left(\mathbb{R}^{N}\right)$ :

$$
\frac{\mathrm{d}}{\mathrm{d} t} \int_{\mathbb{R}^{N}} \Psi\left(u_{r, n}\right) \mathcal{X}_{R} \mathrm{~d} x=\int_{\mathbb{R}^{N}} \mathcal{L}^{\mu_{r}}\left[\varphi_{n}\left(u_{r, n}\right)\right] \Psi^{\prime}\left(u_{r, n}\right) \mathcal{X}_{R} \mathrm{~d} x .
$$

We integrate in time from $t=0$ to $t=\tau$, and use that $u_{r, n} \in$ $C\left([0, T] ; L_{\mathrm{loc}}^{1}\left(\mathbb{R}^{N}\right)\right)\left(\mathrm{cf}\right.$. Theorem 4.5) and $\mathcal{X}_{R} \in C_{\mathrm{c}}^{\infty}\left(\mathbb{R}^{N}\right)$ to obtain

$$
\begin{aligned}
& \int_{\mathbb{R}^{N}} \Psi\left(u_{r, n}(x, \tau)\right) \mathcal{X}_{R}(x) \mathrm{d} x-\int_{\mathbb{R}^{N}} \Psi\left(u_{0}(x)\right) \mathcal{X}_{R}(x) \mathrm{d} x \\
& =\int_{0}^{\tau} \int_{\mathbb{R}^{N}} \mathcal{L}^{\mu_{r}}\left[\varphi_{n}\left(u_{r, n}(\cdot, t)\right)\right](x) \Psi^{\prime}\left(u_{r, n}(x, t)\right) \mathcal{X}_{R}(x) \mathrm{d} x \mathrm{~d} t .
\end{aligned}
$$

Since $\Psi \in W^{1, \infty}(\mathbb{R})$ and $\Psi(0)=0,|\Psi(w)| \leq\left\|\Psi^{\prime}(w)\right\|_{L^{\infty}}|w| \in L^{1}$ for $w=$ $u_{r, n}, u_{0}$. Moreover, since $u_{r, n}, \varphi_{n}\left(u_{r, n}\right)$ and hence also $\mathcal{L}^{\mu_{r}}\left[\varphi_{n}\left(u_{r, n}\right)\right]$ is integrable, we get $\left|\mathcal{L}^{\mu_{r}}\left[\varphi_{n}\left(u_{r, n}\right)\right] \Psi^{\prime}\left(u_{r, n}\right) \mathcal{X}_{R}\right| \in L^{1}\left(\mathbb{R}^{N} \times(0, \tau)\right)$. Then Lebesgue's dominated convergence theorem can be used on both sides of (4.6) as $R \rightarrow \infty$ to complete the proof.

Corollary 4.9 (Energy estimate). Let $\Phi_{n}(w):=\int_{0}^{w} \varphi_{n}(\xi) \mathrm{d} \xi$. Under the assumptions of Proposition 4.7 .

$$
\int_{\mathbb{R}^{N}} \Phi_{n}\left(u_{r, n}(x, \tau)\right) \mathrm{d} x+\left|\varphi_{n}\left(u_{r, n}\right)\right|_{\tau, E_{\mu_{r}}}^{2}=\int_{\mathbb{R}^{N}} \Phi_{n}\left(u_{0}(x)\right) \mathrm{d} x .
$$

In particular,

$$
\left|\varphi_{n}\left(u_{r, n}\right)\right|_{\tau, E_{\mu_{r}}} \leq \int_{\mathbb{R}^{N}} \Phi_{n}\left(u_{0}(x)\right) \mathrm{d} x \leq\left\|\varphi_{n}\left(u_{0}\right)\right\|_{L^{\infty}\left(\mathbb{R}^{N}\right)}\left\|u_{0}\right\|_{L^{1}\left(\mathbb{R}^{N}\right)}<\infty .
$$

Proof. We observe that $\Phi_{n}: \mathbb{R} \rightarrow \mathbb{R}$ is $C^{1}$ and $\Phi_{n}(0)=0$. Moreover, $\Phi_{n}^{\prime}(w)=$ $\varphi_{n}(w)$ which is bounded when $w=: u_{r, n}, u_{0} \in L^{\infty}$ by $\left(\mathrm{A}_{\varphi}\right)$ and (4.2). Hence, $\Phi_{n}$ is Lipschitz, and thus, we can replace $\Psi$ by $\Phi_{n}$ in Proposition 4.7 to get

$$
\begin{aligned}
& \int_{\mathbb{R}^{N}} \Phi_{n}\left(u_{r, n}(x, \tau)\right) \mathrm{d} x-\int_{0}^{\tau} \int_{\mathbb{R}^{N}} \varphi_{n}\left(u_{r, n}(x, t)\right) \mathcal{L}^{\mu_{r}}\left[\varphi_{n}\left(u_{r, n}(\cdot, t)\right)\right](x) \mathrm{d} x \mathrm{~d} t \\
& =\int_{\mathbb{R}^{N}} \Phi_{n}\left(u_{0}(x)\right) \mathrm{d} x .
\end{aligned}
$$

Since (4.2) hold and $\mathcal{L}^{\mu_{r}}\left[\varphi\left(u_{r, n}\right)\right]$ is integrable, we conclude the first part by Lemma 4.3 (take $\left.f=\varphi_{n}\left(u_{r, n}\right)=g\right)$. For the last part, we use that $\Phi_{n}\left(u_{0}\right)=$ $\left|\Phi_{n}\left(u_{0}\right)\right| \leq\left\|\Phi_{n}^{\prime}\left(u_{0}\right)\right\|_{L^{\infty}}\left|u_{0}\right|$, and hence, since $\Phi_{n} \geq 0$,

$$
\left|\varphi_{n}\left(u_{r, n}\right)\right|_{\tau, E_{\mu_{r}}}^{2} \leq \int_{\mathbb{R}^{N}} \Phi_{n}\left(u_{0}(x)\right) \mathrm{d} x \leq\left\|\varphi_{n}\left(u_{0}\right)\right\|_{L^{\infty}}\left\|u_{0}\right\|_{L^{1}}
$$

which completes the proof. 
Lemma 4.10 (General Stroock-Varopoulos). Assume $\left(\mathrm{A}_{\lambda 1}\right), Q, R, S \in C^{1}(\mathbb{R})$, $\left(S^{\prime}\right)^{2} \leq Q^{\prime} R^{\prime}$, and $|Q(\psi)|_{T, E_{\lambda}},|R(\psi)|_{T, E_{\lambda}}<\infty$ for some $\psi: Q_{T} \rightarrow \mathbb{R}$. Then

$$
\int_{0}^{T} \mathcal{E}_{\lambda}[Q(\psi(\cdot, t)), R(\psi(\cdot, t))] \mathrm{d} t \geq|S(\psi)|_{T, E_{\lambda}} .
$$

Proof. Assume without loss of generality that $b>a$. By the Fundamental theorem of calculus, Cauchy-Schwartz' inequality, and $Q^{\prime} R^{\prime} \geq\left(S^{\prime}\right)^{2}$, we obtain

$$
\begin{aligned}
& (Q(b)-Q(a))(R(b)-R(a))=\int_{a}^{b}\left(\sqrt{Q^{\prime}(t)}\right)^{2} \mathrm{~d} t \int_{a}^{b}\left(\sqrt{R^{\prime}(t)}\right)^{2} \mathrm{~d} t \\
& \geq\left(\int_{a}^{b} \sqrt{Q^{\prime}(t) R^{\prime}(t)} \mathrm{d} t\right)^{2} \geq\left(\int_{a}^{b} S^{\prime}(t) \mathrm{d} t\right)^{2}=(S(b)-S(a))^{2} .
\end{aligned}
$$

By the definition of $\mathcal{E}_{\lambda}$ and $|\cdot|_{T, E_{\lambda}}$, the result follows.

Remark 4.11. (a) See Proposition 4.11 in [17] for a similar result.

(b) Observe that the same lemma holds for a nonnegative even Radon measure $\nu$ with $\nu\left(\mathbb{R}^{N}\right)<\infty$ under the simplified assumption $Q(\psi) \in L^{p}\left(Q_{T}\right)$ and $R(\psi) \in L^{q}\left(Q_{T}\right)$ with $1 \leq p, q \leq \infty$ and $\frac{1}{p}+\frac{1}{q}=1$.

Corollary 4.12 ( $L^{p}$-bound). Let $\Lambda(\xi)=|\xi|^{p}$ and $\Xi_{n}(w)=\int_{0}^{w} \sqrt{\Lambda^{\prime \prime}(\xi) \varphi_{n}^{\prime}(\xi)} \mathrm{d} \xi$. Under the assumptions of Proposition 4.7 and $p \in(1, \infty)$,

$$
\int_{\mathbb{R}^{N}}\left|u_{r, n}(x, \tau)\right|^{p} \mathrm{~d} x+\left|\Xi_{n}\left(u_{r, n}\right)\right|_{\tau, E_{\mu_{r}}}^{2} \mathrm{~d} t \leq \int_{\mathbb{R}^{N}}\left|u_{0}(x)\right|^{p} \mathrm{~d} x
$$

In particular,

$$
\int_{\mathbb{R}^{N}}\left|u_{r, n}(x, \tau)\right|^{p} \mathrm{~d} x \leq \int_{\mathbb{R}^{N}}\left|u_{0}(x)\right|^{p} \mathrm{~d} x<\infty .
$$

Remark 4.13. The above result also ensures that $\left|\Xi_{n}\left(u_{r, n}\right)\right|_{\tau, E_{\mu_{r}}}^{2}$ is uniformly bounded in $r$ and $n$.

Proof. Observe that $u_{r, n} \in L^{p}\left(Q_{T}\right)$ for $p \in(1, \infty)$ by standard interpolation in $L^{p}$-spaces.

Case 1: $p \in[2, \infty)$. The function $\Lambda$ is convex, $\Lambda \in W_{\text {loc }}^{1, \infty}(\mathbb{R})$, and $\Lambda(0)=$ 0 . That is, we can replace $\Psi$ by $\Lambda$ in Proposition 4.7 to get

$$
\begin{aligned}
& \int_{\mathbb{R}^{N}} \Lambda\left(u_{r, n}(x, \tau)\right) \mathrm{d} x-\int_{0}^{\tau} \int_{\mathbb{R}^{N}} \Lambda^{\prime}\left(u_{r, n}(x, t)\right) \mathcal{L}^{\mu_{r}}\left[\varphi_{n}\left(u_{r, n}(\cdot, t)\right)\right](x) \mathrm{d} x \mathrm{~d} t \\
& =\int_{\mathbb{R}^{N}} \Lambda\left(u_{0}(x)\right) \mathrm{d} x
\end{aligned}
$$


Note that $\Lambda^{\prime}(\xi)=p|\xi|^{p-2} \xi$ and $\Lambda^{\prime \prime}(\xi)=p(p-1)|\xi|^{p-2}$. Since $\Lambda^{\prime} \in W_{\text {loc }}^{1, \infty}(\mathbb{R})$, $u_{r, n} \in L^{2}\left(Q_{T}\right) \cap L^{\infty}\left(Q_{T}\right)$, and (4.2) holds, $g:=\Lambda^{\prime}\left(u_{r, n}(\cdot, t)\right) \in L^{2}\left(\mathbb{R}^{N}\right)$ and $f:=\varphi_{n}\left(u_{r, n}(\cdot, t)\right) \in L^{2}\left(\mathbb{R}^{N}\right)$. By Lemma 4.3,

$$
-\int_{0}^{\tau} \int_{\mathbb{R}^{N}} \Lambda^{\prime}\left(u_{r, n}\right) \mathcal{L}^{\mu_{r}}\left[\varphi_{n}\left(u_{r, n}\right)\right] \mathrm{d} x \mathrm{~d} t=\int_{0}^{\tau} \mathcal{E}_{\mu_{r}}\left[\Lambda^{\prime}\left(u_{r, n}\right), \varphi_{n}\left(u_{r, n}\right)\right] \mathrm{d} t .
$$

Then by Lemma 4.10 and Remark 4.11 (b) (take $Q:=\Lambda^{\prime}$ and $R:=\varphi_{n}$ ),

$$
\int_{0}^{\tau} \mathcal{E}_{\mu_{r}}\left[\Lambda^{\prime}\left(u_{r, n}(\cdot, t)\right), \varphi_{n}\left(u_{r, n}(\cdot, t)\right)\right] \mathrm{d} t \geq\left|\Xi_{n}\left(u_{r, n}\right)\right|_{\tau, E_{\mu_{r}}}^{2} \geq 0,
$$

since $\Xi_{n}$ satisfies $\left(\Xi_{n}^{\prime}\right)^{2} \leq \Lambda^{\prime \prime} \varphi_{n}^{\prime}$. Hence the corollary follows by (4.7).

CASE 2: $p \in(1,2)$. We follow the idea of the proof of Corollary 5.12 in 9 . For each $\delta>0$, consider the function $\Lambda_{\delta}$ such that

$$
\Lambda_{\delta}(0)=\Lambda_{\delta}^{\prime}(0)=0 \quad \text { and } \quad \Lambda_{\delta}^{\prime \prime}(\xi)=p(p-1)\left(\left(\delta^{2}+\xi^{2}\right)^{\frac{p-2}{2}}-\delta^{p-2}\right) .
$$

Note that $0 \leq \Lambda_{\delta}^{\prime \prime}(\xi) \leq p(p-1)|\xi|^{p-2}$, and then,

$$
\left|\Lambda_{\delta}^{\prime}(\xi)\right|=\left|\int_{0}^{\xi} \Lambda_{\delta}^{\prime \prime}(s) \mathrm{d} s\right| \leq p|\xi|^{p-1} \quad \text { and } \quad\left|\Lambda_{\delta}(\xi)\right| \leq\left|\int_{0}^{\xi} \Lambda_{\delta}^{\prime}(s) \mathrm{d} s\right| \leq|\xi|^{p} .
$$

Since $g:=\Lambda_{\delta}^{\prime}\left(u_{r, n}(\cdot, t)\right) \in L^{\infty}\left(\mathbb{R}^{N}\right)$ and $f:=\varphi_{n}\left(u_{r, n}(\cdot, t)\right) \in L^{1}\left(\mathbb{R}^{N}\right)$, we get - by following the calculations in Case 1 - that

$$
\int_{\mathbb{R}^{N}} \Lambda_{\delta}\left(u_{r, n}(x, \tau)\right) \mathrm{d} x+\left|\Xi_{n, \delta}\left(u_{r, n}\right)\right|_{\tau, E_{\mu_{r}}}^{2} \leq \int_{\mathbb{R}^{N}} \Lambda_{\delta}\left(u_{0}(x)\right) \mathrm{d} x
$$

with

$$
\Xi_{n, \delta}\left(u_{r, n}\right)=\int_{0}^{u_{r, n}} \sqrt{\Lambda_{\delta}^{\prime \prime}(\xi) \varphi_{n}^{\prime}(\xi)} \mathrm{d} \xi \geq 0 .
$$

By a direct argument, using $\Lambda_{\delta}^{\prime \prime}, \Lambda^{\prime \prime}, \varphi_{n}^{\prime} \geq 0$ and Cauchy-Schwartz's inequality, we obtain

$$
\begin{aligned}
\left|\Xi_{n, \delta}\left(u_{r, n}\right)-\Xi_{n}\left(u_{r, n}\right)\right| & \leq \int_{0}^{u_{r, n}} \sqrt{\left|\Lambda_{\delta}^{\prime \prime}(\xi)-\Lambda^{\prime \prime}(\xi)\right|} \sqrt{\varphi_{n}^{\prime}(\xi)} \mathrm{d} \xi \\
& \leq \sqrt{\int_{0}^{u_{r, n}}\left|\Lambda_{\delta}^{\prime \prime}(\xi)-\Lambda^{\prime \prime}(\xi)\right| \mathrm{d} \xi} \sqrt{\int_{0}^{u_{r, n}} \varphi_{n}^{\prime}(\xi) \mathrm{d} \xi} \\
& \leq\left\|\varphi_{n}\left(u_{r, n}\right)\right\|_{L^{\infty}\left(Q_{T}\right)}^{\frac{1}{2}} \sqrt{\int_{0}^{u_{r, n}}\left|\Lambda_{\delta}^{\prime \prime}(\xi)-\Lambda^{\prime \prime}(\xi)\right| \mathrm{d} \xi} .
\end{aligned}
$$

Since the integrand in the last inequality is dominated by $2 p(p-1)|\xi|^{p-2}$ which integrates to $2 p\left|u_{r, n}\right|^{p-2} u_{r, n}$, we use Lebesgue's dominated convergence theorem to conclude that $\Xi_{n, \delta} \rightarrow \Xi_{n}$ as $\delta \rightarrow 0^{+}$. Taking the limit as $\delta \rightarrow$ $0^{+}$in (4.8), by using Fatou's lemma on the left-hand side and Lebesgue's dominated convergence theorem $\left(\left|\Lambda_{\delta}\left(u_{0}(x)\right)\right| \leq\left|u_{0}(x)\right|^{p}\right)$ on the right-hand side, the corollary follows. 
Remark 4.14. Observe that by (4.9),

$$
\left|\Xi_{n, \delta}\left(u_{r, n}\right)\right| \leq p\left\|\varphi_{n}\left(u_{r, n}\right)\right\|_{L^{\infty}\left(Q_{T}\right)}^{\frac{1}{2}}\left\|u_{r, n}\right\|_{L^{\infty}\left(Q_{T}\right)}^{\frac{p-1}{2}}<\infty,
$$

and similarly for $\Xi_{n}\left(u_{r, n}\right)$. Hence, both are well-defined for all $p \in(1, \infty)$.

The existence of a distributional solution of (1.3)-(1.4) with finite energy (cf. Theorem 2.13) will follow from the following compactness theorem:

Theorem 4.15 (Compactness). Assume $\left(\mathrm{A}_{\varphi}\right),\left(\mathrm{A}_{\mu}\right)$, and $\left(\mathrm{A}_{u_{0}}\right)$. Let $\left\{u_{r, n}\right\}_{r, n \in \mathbb{N}}$ be a sequence of distributional solutions of (4.3)-(4.4). Then there exists a subsequence $\left\{u_{r_{j}, n_{j}}\right\}_{j \in \mathbb{N}}$ and $a u \in C\left([0, T] ; L_{\mathrm{loc}}^{1}\left(\mathbb{R}^{N}\right)\right)$ such that

$$
u_{r_{j}, n_{j}} \rightarrow u \quad \text { in } \quad C\left([0, T] ; L_{\mathrm{loc}}^{1}\left(\mathbb{R}^{N}\right)\right) \quad \text { as } \quad j \rightarrow \infty .
$$

Moreover, $u \in L^{1}\left(Q_{T}\right) \cap L^{\infty}\left(Q_{T}\right) \cap C\left([0, T] ; L_{\text {loc }}^{1}\left(\mathbb{R}^{N}\right)\right)$ is a distributional solution of (1.3) -(1.4).

Remark 4.16. We have that $\|u\|_{L^{1} / L^{\infty}} \leq\left\|u_{0}\right\|_{L^{1 / L^{\infty}}}$ by Fatou's lemma and Remark 4.4 (the limit of a uniformly bounded sequence is uniformly bounded by the same bound).

Proof. Observe that the sequence $\left\{u_{r_{j}, n_{j}}\right\}_{j \in \mathbb{N}}$ enjoy $L^{1}-, L^{\infty}$-bounds, and time regularity by Remark 4.4, and that these bounds are independent of $j$ (see Section 4 in [22]).

Moreover, for any $\psi \in C_{\mathrm{c}}^{\infty}\left(\mathbb{R}^{N}\right)$,

$$
\left(\mathcal{L}^{\mu}-\mathcal{L}^{\mu_{r_{j}}}\right)[\psi](x)=\int_{|z| \leq r_{j}}(\psi(x+z)-\psi(x)-z \cdot D \psi(x)) \mu(\mathrm{d} z),
$$

and hence, $\mathcal{L}^{\mu_{r_{j}}}[\psi] \rightarrow \mathcal{L}^{\mu}[\psi]$ in $L^{1}\left(\mathbb{R}^{N}\right)$ as $r_{j} \rightarrow 0^{+}$by Lebesgue's dominated convergence theorem. We also have,

$$
\sup _{r_{j}>0} \int_{|z|>0} \min \left\{|z|^{2}, 1\right\} \mathrm{d} \mu_{r_{j}}(z) \leq \int_{|z|>0} \min \left\{|z|^{2}, 1\right\} \mathrm{d} \mu(z)<\infty,
$$

and $\varphi_{n_{j}} \rightarrow \varphi$ locally uniformly as $n_{j} \rightarrow \infty$ by (4.2). Thus, we are in the setting of Theorem 2.12 in [22] and the result follows.

We are now ready to prove Theorems 2.13, 2.19, and 2.21,

Proof of Theorem 2.13 (Existence 1). In light of Theorem4.15, it only remains to prove that the limit $u$ is such that $\varphi(u) \in L^{2}\left(0, T ; E_{\mu}\left(\mathbb{R}^{N}\right)\right)$. Recall that $\Phi_{n_{j}}(w)=\int_{0}^{w} \varphi_{n_{j}}(\xi) \mathrm{d} \xi$ and $\Phi(w)=\int_{0}^{w} \varphi(\xi) \mathrm{d} \xi$. Now,

$$
\begin{aligned}
\left|\int_{\mathbb{R}^{N}} \Phi\left(u_{0}\right) \mathrm{d} x-\int_{\mathbb{R}^{N}} \Phi_{n_{j}}\left(u_{0}\right) \mathrm{d} x\right| & \leq \int_{\mathbb{R}^{N}}\left|\Phi\left(u_{0}\right)-\Phi_{n_{j}}\left(u_{0}\right)\right| \mathrm{d} x \\
& \leq \int_{\mathbb{R}^{N}} \int_{0}^{u_{0}}\left|\varphi(\xi)-\varphi_{n_{j}}(\xi)\right| \mathrm{d} \xi \mathrm{d} x \\
& \leq\left\|u_{0}\right\|_{L^{1}} \sup _{|\xi| \leq\left\|u_{0}\right\|_{L^{\infty}}}\left|\varphi(\xi)-\varphi_{n_{j}}(\xi)\right|,
\end{aligned}
$$


and since $\varphi_{n_{j}} \rightarrow \varphi$ locally uniformly, $\lim _{n_{j} \rightarrow \infty} \int_{\mathbb{R}^{N}} \Phi_{n_{j}}\left(u_{0}\right) \mathrm{d} x=\int_{\mathbb{R}^{N}} \Phi\left(u_{0}\right) \mathrm{d} x$. Observe also that by Theorem 4.15 (and Remark 4.4), (4.2), and the proof of Theorem 2.6 in [22], we can take a further subsequence to get that $\varphi_{n_{j}}\left(u_{r_{j}, n_{j}}\right) \rightarrow$ $\varphi(u)$ a.e. in $Q_{T}$ as $j \rightarrow \infty$.

For any $R \geq r_{j}>0,\left|\varphi_{n_{j}}\left(u_{r_{j}, n_{j}}\right)\right|_{E_{\mu_{R}}}^{2} \leq\left|\varphi_{n_{j}}\left(u_{r_{j}, n_{j}}\right)\right|_{E_{\mu_{r_{j}}}}^{2}$, and thus, by the second part of Corollary 4.9 ,

$$
\int_{0}^{\tau}\left|\varphi_{n_{j}}\left(u_{r_{j}, n_{j}}\right)\right|_{E_{\mu_{R}}}^{2} \mathrm{~d} t \leq \int_{\mathbb{R}^{N}} \Phi_{n_{j}}\left(u_{0}\right) \mathrm{d} x .
$$

Taking the limit as $j \rightarrow \infty$, we obtain, by Fatou's lemma, the above calculations, and the estimate $\Phi\left(u_{0}\right) \leq\left\|\varphi\left(u_{0}\right)\right\|_{L^{\infty}}\left|u_{0}\right|$, that

$$
\int_{0}^{\tau}|\varphi(u)|_{E_{\mu_{R}}}^{2} \mathrm{~d} t \leq \int_{\mathbb{R}^{N}} \Phi\left(u_{0}\right) \mathrm{d} x \leq\left\|\varphi\left(u_{0}\right)\right\|_{L^{\infty}}\left\|u_{0}\right\|_{L^{1}} .
$$

Another application of Fatou's lemma, as $R \rightarrow 0^{+}$, and the choice $\tau=T$ yield

$$
|\varphi(u)|_{T, E_{\mu}} \leq\|\varphi(u)\|_{L^{\infty}\left(\mathbb{R}^{N}\right)}\left\|u_{0}\right\|_{L^{1}\left(\mathbb{R}^{N}\right)}<\infty .
$$

The proof is complete.

By Theorem 2.15, we know that any subsequence of $\left\{u_{r, n}\right\}_{r, n \in \mathbb{N}}$ converges to the same limit, and hence, the whole sequence converges since it is bounded by Remark 4.4. Let us then continue with the proof of the energy and $L^{p_{-}}$ estimates for the distributional solution of (1.3)-(1.4).

Proof of Theorem 2.19 (Energy inequality). By Remark 4.16,

$$
\begin{aligned}
& \left|\Phi(u(x, t))-\Phi_{n}\left(u_{r, n}(x, t)\right)\right| \\
& \leq \sup _{|\xi| \leq 2\left\|u_{0}\right\|_{L^{\infty}}}|\varphi(\xi)|\left|u(x, t)-u_{r, n}(x, t)\right|+\left\|u_{0}\right\|_{L^{\infty}} \sup _{|\xi| \leq\left\|u_{0}\right\|_{L^{\infty}}}\left|\varphi(\xi)-\varphi_{n}(\xi)\right| .
\end{aligned}
$$

Since $\varphi_{n} \rightarrow \varphi$ locally uniformly and we can find a subsequence of $\left\{u_{r, n}\right\}_{r, n}$ such that $u_{r_{j}, n_{j}} \rightarrow u$ a.e. in $Q_{T}$ as $j \rightarrow \infty$ by Theorem 4.15, $\Phi_{n_{j}}\left(u_{r_{j}, r_{j}}(x, t)\right) \rightarrow$ $\Phi(u(x, t))$ pointwise a.e. in $Q_{T}$ as $j \rightarrow \infty$. The conclusion then follows by Corollary 4.9, Fatou's lemma, and the proof of Theorem 2.13.

Proof of Theorem 2.21 ( $L^{p}$-bounds). By Fatou's lemma and Theorem4.15, we can take the limit as $j \rightarrow \infty$ (since $u_{r_{j}, n_{j}} \rightarrow u$ a.e. in $Q_{T}$ as $j \rightarrow \infty$ by considering a further subsequence in Theorem 4.15) in the second estimate in Corollary 4.12 to obtain the result. The cases $p=1$ and $p=\infty$ are explained in Remark 4.16] 
Acknowledgments. E. R. Jakobsen was supported by the Toppforsk (research excellence) project Waves and Nonlinear Phenomena (WaNP), grant no. 250070 from the Research Council of Norway. F. del Teso was supported by the FPU grant AP2010-1843 and the grants MTM2011-24696 and MTM201452240-P from the Ministry of Education, Culture and Sports, Spain, the BERC 2014-2017 program from the Basque Government, and BCAM Severo Ochoa excellence accreditation SEV-2013-0323 from Spanish Ministry of Economy and Competitiveness (MINECO). We would like to thank Stefano Lisini and Giampiero Palatucci for fruitful discussions on homogeneous fractional Sobolev spaces.

\section{A. Proof of Theorem 2.6 (a)}

Obviously $X \cap L^{2}\left(Q_{T}\right) \subset L^{2}\left(0, T ; E_{\lambda}\left(\mathbb{R}^{N}\right)\right) \cap L^{\infty}\left(Q_{T}\right) \cap L^{2}\left(Q_{T}\right)$, and we must show the opposite inclusion: Any

$$
f \in L^{2}\left(0, T ; E_{\lambda}\left(\mathbb{R}^{N}\right)\right) \cap L^{\infty}\left(Q_{T}\right) \cap L^{2}\left(Q_{T}\right)
$$

belongs to $X \cap L^{2}\left(Q_{T}\right)$. To do so, we must prove that $f$ can be suitably approximated by functions in $C_{\mathrm{c}}^{\infty}\left(\mathbb{R}^{N} \times[0, T)\right)$. We will now explain how to build such an approximation.

Let $\delta>0$ and $g_{\delta}: \mathbb{R}^{N+1} \rightarrow \mathbb{R}$ be defined by

$$
g_{\delta}(x, t):=f(x, t) \mathbf{1}_{[2 \delta, T-3 \delta]}(t)
$$

and mollify $g_{\delta}$ to get

$$
G_{\delta}(x, t):=g_{\delta} *_{x, t} \rho_{\delta}(x, t)=\iint_{\mathbb{R}^{N+1}} g_{\delta}(y, s) \rho_{\delta}(x-y, t-s) \mathrm{d} y \mathrm{~d} s
$$

where $\rho_{\delta}$ is defined by $\rho_{\delta}(\sigma, \tau):=\frac{1}{\delta^{N+1}} \rho\left(\frac{\sigma}{\delta}, \frac{\tau}{\delta}\right)$ for a fixed $0 \leq \rho \in C_{\mathrm{c}}^{\infty}\left(\mathbb{R}^{N+1}\right)$ satisfying supp $\rho \subseteq \bar{B}(0,1) \times[-1,1], \rho(\sigma, \tau)=\rho(-\sigma,-\tau)$, and $\iint \rho=1$. Note that $\left|g_{\delta}\right| \leq|f|$ and $G_{\delta} \in C^{\infty}\left(\mathbb{R}^{N+1}\right)$ with support in $\mathbb{R}^{N} \times[\delta, T-2 \delta]$.

Lemma A.1. Assume $\left(\mathrm{A}_{\lambda 0}\right),\left(\mathrm{A}_{\lambda 1}{ }^{\prime}\right),\left(\mathrm{A}_{\lambda 2}\right)$, and $f \in L^{2}\left(0, T ; E_{\lambda}\left(\mathbb{R}^{N}\right)\right) \cap$ $L^{\infty}\left(Q_{T}\right) \cap L^{2}\left(Q_{T}\right)$.

(a) $G_{\delta} \in C_{0}\left(\mathbb{R}^{N+1}\right)$.

(b) $\left|G_{\delta}\right|_{T, E_{\lambda}}^{2} \leq C|f|_{T, E_{\lambda}}^{2}+4\|f\|_{L^{2}\left(Q_{T}\right)}^{2}\left\|\Pi_{\lambda}\right\|_{L^{\infty}\left(\mathbb{R}^{N}\right)}$ for some constant $C \geq 0$.

Remark A.2. If $\lambda$ is globally shift-bounded, that is, we replace the statement " $B \subset B(0,1) \backslash\{0\}$ " with "for all $B \in \mathbb{R}^{N} \backslash\{0\}$ " in $\left(\mathrm{A}_{\lambda 1}\right.$ '), then in (b) we get

$$
\left|G_{\delta}\right|_{T, E_{\lambda}}^{2} \leq C|f|_{T, E_{\lambda}}^{2} .
$$

In this case, we do not have to assume $\Pi_{\lambda} \in L^{\infty}\left(\mathbb{R}^{N}\right)$ and $\left(\mathrm{A}_{\lambda 2}\right)$. 
Proof. (a) Since the Fourier transforms of $g_{\delta}$ and $\rho_{\delta}$ are both in $L^{2}\left(\mathbb{R}^{N+1}\right)$, the properties of the Fourier transform and Hölder's inequality yield

$$
\mathcal{F}\left(G_{\delta}\right)=\mathcal{F}\left(g_{\delta} * \rho_{\delta}\right)=\mathcal{F}\left(g_{\delta}\right) \mathcal{F}\left(\rho_{\delta}\right) \in L^{1}\left(\mathbb{R}^{N+1}\right) .
$$

The result then follows by the Riemann-Lebesgue lemma which gives that $G_{\delta}=\mathcal{F}^{-1}\left(\mathcal{F}\left(G_{\delta}\right)\right) \in C_{0}\left(\mathbb{R}^{N+1}\right)$.

(b) The proof is a straightforward adaptation of the proof of Lemma 2.2 in [36] and the estimate $\left|g_{\delta}\right| \leq|f|$.

Next, we recall a useful truncation from [36]: Let $T_{\delta}: \mathbb{R} \rightarrow \mathbb{R}$ be defined by

$$
T_{\delta}(x):=\min \left\{\max \left\{-\frac{1}{\delta}, x-\min \{\max \{-\delta, x\}, \delta\}\right\}, \frac{1}{\delta}\right\} \quad \text { for all } \quad x \in \mathbb{R} .
$$

Observe that for all $x, y \in \mathbb{R}$

$$
\left|T_{\delta}(x)\right| \leq|x|, \quad\left|T_{\delta}(x)-T_{\delta}(y)\right| \leq|x-y|, \text { and } \quad T_{\delta}(x) \rightarrow x \text { as } \delta \rightarrow 0^{+} .
$$

We can now define a $C_{\mathrm{c}}^{\infty}$-approximation of $f$ :

$$
w_{\delta}(x, t):=T_{\delta}\left[G_{\delta}\right] *{ }_{x, t} \rho_{\delta}(x, t) .
$$

Lemma A.3. Assume $\left(\mathrm{A}_{\lambda 0}\right),\left(\mathrm{A}_{\lambda 1}{ }^{\prime}\right),\left(\mathrm{A}_{\lambda 2}\right)$, and $f \in L^{2}\left(0, T ; E_{\lambda}\left(\mathbb{R}^{N}\right)\right) \cap$ $L^{\infty}\left(Q_{T}\right) \cap L^{2}\left(Q_{T}\right)$. Then:

(a) $w_{\delta} \in C_{\mathrm{c}}^{\infty}\left(\mathbb{R}^{N+1}\right)$ and $\operatorname{supp} w_{\delta} \subset \mathbb{R}^{N} \times[0, T-\delta]$.

(b) $\left\|w_{\delta}-f\right\|_{L^{2}\left(Q_{T}\right)} \rightarrow 0$ as $\delta \rightarrow 0^{+}$.

(c) For some $K \geq 0,\left\|w_{\delta}\right\|_{L^{2}\left(Q_{T}\right)}+\left\|w_{\delta}\right\|_{L^{\infty}\left(Q_{T}\right)}+\left|w_{\delta}\right|_{T, E_{\lambda}} \leq K$ for all $\delta>0$.

Remark A.4. If $f \in L^{p}$ for some $p \in[1, \infty)$, similar arguments show that (b) can be replaced by $\left\|w_{\delta}-f\right\|_{L^{p}\left(Q_{T}\right)} \rightarrow 0$ as $\delta \rightarrow 0^{+}$. Moreover, if the measure $\lambda$ is globally shift-bounded, then we can relax assumption $\left(\mathrm{A}_{\lambda 1}{ }^{\prime}\right)$ as in Remark A.2. and replace the previous uniform bound of $\left|w_{\delta}\right|_{T, E_{\lambda}}$ by $C|f|_{T, E_{\lambda}}$ in (c).

Proof. (a) Since $G_{\delta}$ vanishes at infinity, $T_{\delta}\left[G_{\delta}\right]$ has compact support, and therefore $w_{\delta}:=T_{\delta}\left[G_{\delta}\right] *_{x, t} \rho_{\delta} \in C_{\mathrm{c}}^{\infty}\left(\mathbb{R}^{N+1}\right)$. Moreover, $\operatorname{supp} T_{\delta}\left[G_{\delta}\right] \subset \mathbb{R}^{N} \times$ $[\delta, T-\delta]$ and hence supp $w_{\delta} \subset \mathbb{R}^{N} \times[0, T-\delta]$. As a consequence, $w_{\delta} \subset$ $C_{\mathrm{c}}^{\infty}\left(\mathbb{R}^{N} \times[0, T)\right)$.

(b) Note that $\left|T_{\delta}\left[G_{\delta}\right]\right|^{2} \leq\left|G_{\delta}\right|^{2}$ by (A.2), $\left\|G_{\delta}\right\|_{L^{2}} \leq\left\|g_{\delta}\right\|_{L^{2}}$, and $\left|g_{\delta}\right|^{2} \leq|f|^{2}$, and thus, all these functions are in $L^{2}$. Hence,

$$
\left\|w_{\delta}-f\right\|_{L^{2}\left(Q_{T}\right)} \leq\left\|w_{\delta}-G_{\delta}\right\|_{L^{2}\left(Q_{T}\right)}+\left\|G_{\delta}-g_{\delta}\right\|_{L^{2}\left(Q_{T}\right)}+\left\|g_{\delta}-f\right\|_{L^{2}\left(Q_{T}\right)}
$$


and by the properties of mollifiers and (A.2),

$$
\begin{aligned}
\left\|w_{\delta}-G_{\delta}\right\|_{L^{2}} & \leq\left\|T_{\delta}\left[G_{\delta}\right] *_{x, t} \rho_{\delta}-T_{\delta}\left[g_{\delta}\right] *_{x, t} \rho_{\delta}\right\|_{L^{2}}+\left\|T_{\delta}\left[g_{\delta}\right] *_{x, t} \rho_{\delta}-G_{\delta}\right\|_{L^{2}} \\
& \leq\left\|T_{\delta}\left[G_{\delta}\right]-T_{\delta}\left[g_{\delta}\right]\right\|_{L^{2}}+\left\|T_{\delta}\left[g_{\delta}\right]-g_{\delta}\right\|_{L^{2}} \\
& \leq\left\|G_{\delta}-g_{\delta}\right\|_{L^{2}}+\left\|T_{\delta}\left[g_{\delta}\right]-g_{\delta}\right\|_{L^{2}} .
\end{aligned}
$$

Finally, we can use Lebesgue's dominated convergence theorem $\left(\left|T_{\delta}\left[g_{\delta}\right]\right|^{2} \leq\right.$ $\left|g_{\delta}\right|^{2}$ by (A.2) and $\left|g_{\delta}\right|^{2} \leq|f|^{2}$ ) and the properties of mollifiers to conclude.

(c) According to Lemma A.1 (b),

$$
\left|w_{\delta}\right|_{T, E_{\lambda}}^{2} \leq C\left|T_{\delta}\left[G_{\delta}\right]\right|_{T, E_{\lambda}}^{2}+4\left\|T_{\delta}\left[G_{\delta}\right]\right\|_{L^{2}\left(Q_{T}\right)}^{2}\left\|\Pi_{\lambda}\right\|_{L^{\infty}\left(\mathbb{R}^{N}\right)},
$$

and then by (A.2),

$$
\left|T_{\delta}\left[G_{\delta}\right]\right|_{T, E_{\lambda}}^{2} \leq\left|G_{\delta}\right|_{T, E_{\lambda}}^{2} \quad \text { and } \quad\left\|T_{\delta}\left[G_{\delta}\right]\right\|_{L^{2}\left(Q_{T}\right)}^{2} \leq\left\|G_{\delta}\right\|_{L^{2}\left(Q_{T}\right)}^{2} .
$$

So, by another application of Lemma A.1, the properties of mollifiers, $\left|g_{\delta}\right| \leq$ $|f|$, and $\left|g_{\delta}(x, t)-g_{\delta}(y, t)\right| \leq|f(x, t)-f(y, t)|$, we have

$$
\left|w_{\delta}\right|_{T, E_{\lambda}}^{2} \leq C\left(|f|_{T, E_{\lambda}}^{2}+\|f\|_{L^{2}\left(Q_{T}\right)}^{2}\left\|\Pi_{\lambda}\right\|_{L^{\infty}\left(\mathbb{R}^{N}\right)}\right) .
$$

Note also that by part (b), $\left\|w_{\delta}\right\|_{L^{2}\left(Q_{T}\right)} \leq\|f\|_{L^{2}\left(Q_{T}\right)}$, and moreover, by the properties of mollifiers, (A.2), and the definition of $g_{\delta}$,

$$
\left\|w_{\delta}\right\|_{L^{\infty}\left(Q_{T}\right)} \leq\left\|T_{\delta}\left[G_{\delta}\right]\right\|_{L^{\infty}\left(\mathbb{R}^{N+1}\right)} \leq\left\|G_{\delta}\right\|_{L^{\infty}\left(\mathbb{R}^{N+1}\right)} \leq\|f\|_{L^{\infty}\left(Q_{T}\right)} .
$$

This completes the proof.

To prove Theorem 2.6 (a), we will define from $\left\{w_{\delta}\right\}_{\delta>0}$ a $C_{\mathrm{c}}^{\infty}$-sequence that converges also in $|\cdot|_{T, E_{\lambda}}$.

Proof of Theorem 2.6 (a). This proof is an adaptation the proof of Theorem 2.4 in [36. Note that by standard arguments $L^{2}\left(Q_{T}\right) \cap L^{2}\left(0, T ; E_{\lambda}\left(\mathbb{R}^{N}\right)\right)$ is a Hilbert space with inner product $\langle\cdot, \cdot\rangle_{L^{2}\left(Q_{T}\right)}+\int_{0}^{T} \mathcal{E}_{\lambda}[\cdot, \cdot] \mathrm{d} t$. By Lemma A.3 (c) and Banach-Saks' theorem, there is a subsequence $\left\{w_{\delta_{k}}\right\}_{k \in \mathbb{N}}$ such that the Césaro mean of this subsequence converges to some function $\tilde{f} \in L^{2}\left(Q_{T}\right) \cap$ $L^{2}\left(0, T ; E_{\lambda}\left(\mathbb{R}^{N}\right)\right)$ :

$$
\left\|\frac{1}{n} \sum_{k=1}^{n} w_{\delta_{k}}-\tilde{f}\right\|_{L^{2}\left(Q_{T}\right)}^{2}+\left|\frac{1}{n} \sum_{k=1}^{n} w_{\delta_{k}}-\tilde{f}\right|_{T, E_{\lambda}}^{2} \rightarrow 0 \quad \text { as } \quad n \rightarrow \infty .
$$

Let $\phi_{n}=\frac{1}{n} \sum_{k=1}^{n} w_{\delta_{k}}$. Then $\phi_{n} \in C_{\mathrm{c}}^{\infty}\left(\mathbb{R}^{N} \times[0, T)\right)$ and is uniformly bounded in $L^{\infty}\left(Q_{T}\right)$ since $w_{\delta}$ is (cf. Lemma A.3 (c)). By Banach-Alaoglu's theorem, we can take a further subsequence (also denoted $\phi_{n}$ ) such that

$$
\phi_{n} \stackrel{*}{\rightarrow} \bar{f} \quad \text { in } \quad L^{\infty}\left(Q_{T}\right) \quad \text { as } \quad n \rightarrow \infty .
$$


Since $w_{\delta} \rightarrow f$ in $L^{2}\left(Q_{T}\right)$ as $\delta \rightarrow 0^{+}$by Lemma A.3 (b), any subsequence and any Césaro mean of this subsequence converges to $f \in L^{2}\left(Q_{T}\right)$. All three notions of convergence implies distributional convergence, and hence, the result follows since by uniqueness of limits, $f=\tilde{f}=\bar{f}$ in $\mathcal{D}^{\prime}\left(\mathbb{R}^{N} \times[0, T)\right)$ and then a.e.

\section{B. On the spaces $\dot{H}^{\frac{\alpha}{2}}\left(\mathbb{R}^{N}\right)$ and $L^{2}\left(0, T ; \dot{H}^{\frac{\alpha}{2}}\left(\mathbb{R}^{N}\right)\right)$}

In the first part of this section we prove the equivalence between three different definitions of the homogeneous Sobolev space $\dot{H}^{\frac{\alpha}{2}}\left(\mathbb{R}^{N}\right)$ when $N>\alpha$. These results are well-known, but we were unable to find proofs that directly apply to our setting. Then in the second part, we define the parabolic space $L^{2}\left(0, T ; \dot{H}^{\frac{\alpha}{2}}\left(\mathbb{R}^{N}\right)\right)$ and show some of its properties. Note that we do not define this space as a Bochner space, but rather as an iterated $L^{2}-\dot{H}^{\frac{\alpha}{2}}$ space. Our discussion heavily relies on [8], 77, [36], and [33].

In the next section we use these results to prove Theorem 2.6 (b).

Proposition B.1. Assume $\alpha \in(0,2)$ and $N>\alpha$. Let $f \in \mathcal{S}^{\prime}\left(\mathbb{R}^{N}\right)$, a tempered distribution, $\mathcal{F}\{f\}$ its Fourier transform, and

$$
|f|_{\dot{H}^{\frac{\alpha}{2}\left(\mathbb{R}^{N}\right)}}^{2}:=\int_{\mathbb{R}^{N}}|\xi|^{\alpha}|\mathcal{F}\{f\}(\xi)|^{2} \mathrm{~d} \xi<\infty .
$$

The following definitions of $\dot{H}^{\frac{\alpha}{2}}\left(\mathbb{R}^{N}\right)$ are equivalent:

(a) $\dot{H}_{1}^{\frac{\alpha}{2}}\left(\mathbb{R}^{N}\right):=\left\{f \in \mathcal{S}^{\prime}\left(\mathbb{R}^{N}\right): \mathcal{F}\{f\} \in L_{\mathrm{loc}}^{1}\left(\mathbb{R}^{N}\right)\right.$ and $\left.|f|_{\dot{H}^{\frac{\alpha}{2}}\left(\mathbb{R}^{N}\right)}<\infty\right\}$,

(b) $\dot{H}_{2}^{\frac{\alpha}{2}}\left(\mathbb{R}^{N}\right):={\overline{C_{\mathrm{C}}^{\infty}\left(\mathbb{R}^{N}\right)}}^{|\cdot|_{\dot{H}^{\frac{\alpha}{2}}}}{ }_{\left(\mathbb{R}^{N}\right)}$, and

(c) $\dot{H}_{3}^{\frac{\alpha}{2}}\left(\mathbb{R}^{N}\right):=\left\{f \in L^{\frac{2 N}{N-\alpha}}\left(\mathbb{R}^{N}\right):|f|_{\dot{H}^{\frac{\alpha}{2}}\left(\mathbb{R}^{N}\right)}<\infty\right\}$.

Proof. 1) By Propositions 1.34 and 1.37 and Theorem 1.38 in [7, $\dot{H}_{1}^{\frac{\alpha}{2}}\left(\mathbb{R}^{N}\right)$ is a Hilbert space with the norm

$$
|f|_{\dot{H}^{\frac{\alpha}{2}}\left(\mathbb{R}^{N}\right)}=\int_{\mathbb{R}^{N}}|\xi|^{\alpha}|\mathcal{F}\{f\}(\xi)|^{2} \mathrm{~d} \xi=c_{N, \alpha} \int_{\mathbb{R}^{N}} \int_{|z|>0} \frac{|f(x+z)-f(x)|^{2}}{|z|^{N+\alpha}} \mathrm{d} z \mathrm{~d} x,
$$

and $\dot{H}_{1}^{\frac{\alpha}{2}}\left(\mathbb{R}^{N}\right)$ is continuously embedded in $L^{\frac{2 N}{N-\alpha}}\left(\mathbb{R}^{N}\right)$ with

$$
\|f\|_{L^{\frac{2 N}{N-\alpha}}\left(\mathbb{R}^{N}\right)} \leq C|f|_{\dot{H}^{\frac{\alpha}{2}}\left(\mathbb{R}^{N}\right)} .
$$

We also note that $\frac{2 N}{N-\alpha} \in(2, \infty)$ as long as $N>\alpha$. 
2) $\dot{H}_{2}^{\frac{\alpha}{2}}\left(\mathbb{R}^{N}\right) \subset \dot{H}_{1}^{\frac{\alpha}{2}}\left(\mathbb{R}^{N}\right)$ : For any $f \in \dot{H}_{2}^{\frac{\alpha}{2}}\left(\mathbb{R}^{N}\right)$, it is clear that $f$ is a tempered distribution and $|f|_{\dot{H}^{\frac{\alpha}{2}}}\left(\mathbb{R}^{N}\right)<\infty$. Furthermore, $\mathcal{F}\{f\} \in L_{\text {loc }}^{1}\left(\mathbb{R}^{N}\right)$ since for any compact $K \subset \mathbb{R}^{N}$, we use Hölder's inequality to get

$$
\int_{K}|\mathcal{F}\{f\}| \mathrm{d} \xi \leq\left(\int_{\mathbb{R}^{N}}|\xi|^{\alpha}|\mathcal{F}\{f\}|^{2} \mathrm{~d} \xi\right)^{\frac{1}{2}}\left(\int_{K}|\xi|^{-\alpha} \mathrm{d} \xi\right)^{\frac{1}{2}}<\infty .
$$

3) $\dot{H}_{1}^{\frac{\alpha}{2}}\left(\mathbb{R}^{N}\right) \subset \dot{H}_{2}^{\frac{\alpha}{2}}\left(\mathbb{R}^{N}\right)$ : Due to Remark A.4, we can proceed as in Section A (or Theorem 2.4 in [36]): For any $f \in \dot{H}_{1}^{\frac{\alpha}{2}}\left(\mathbb{R}^{N}\right)$, we construct an $C_{\mathrm{c}}^{\infty}$ approximation $w_{\delta}$ satisfying

$$
\left\|w_{\delta}-f\right\|_{L^{\frac{2 N}{N-\alpha}}\left(\mathbb{R}^{N}\right)} \stackrel{\delta \rightarrow 0^{+}}{\longrightarrow} 0 \quad \text { and } \quad\left|w_{\delta}\right|_{\dot{H}^{\frac{\alpha}{2}}\left(\mathbb{R}^{N}\right)} \leq C|f|_{\dot{H}^{\frac{\alpha}{2}}\left(\mathbb{R}^{N}\right)} .
$$

Hence since $\dot{H}_{1}^{\frac{\alpha}{2}}\left(\mathbb{R}^{N}\right)$ is a Hilbert space, Banach-Saks' theorem ensures the existence of a subsequence $\left\{w_{\delta_{k}}\right\}_{k \in \mathbb{N}}$ and $\tilde{f} \in \dot{H}_{1}^{\frac{\alpha}{2}}\left(\mathbb{R}^{N}\right)$ such that

$$
\left|\frac{1}{n} \sum_{k=1}^{n} w_{\delta_{k}}-\tilde{f}\right|_{\dot{H}^{\frac{\alpha}{2}}\left(\mathbb{R}^{N}\right)}^{2} \rightarrow 0 \quad \text { as } \quad n \rightarrow \infty .
$$

By (B.1), these Césaro means converge to $\tilde{f}$ in $L^{\frac{2 N}{N-\alpha}}$. But by (B.3), they also converge to $f$ in $L^{\frac{2 N}{N-\alpha}}$, and hence $f=\tilde{f}$ a.e.

4) $\dot{H}_{3}^{\frac{\alpha}{2}}\left(\mathbb{R}^{N}\right) \subset \dot{H}_{1}^{\frac{\alpha}{2}}\left(\mathbb{R}^{N}\right)$ : Since $f \in L^{\frac{2 N}{N-\alpha}}\left(\mathbb{R}^{N}\right)$, it is a tempered distribution, and $\mathcal{F}\{f\} \in L_{\text {loc }}^{1}\left(\mathbb{R}^{N}\right)$ by $(\overline{B .2})$.

5) $\dot{H}_{1}^{\frac{\alpha}{2}}\left(\mathbb{R}^{N}\right) \subset \dot{H}_{3}^{\frac{\alpha}{2}}\left(\mathbb{R}^{N}\right)$ : This is just a consequence of (B.1).

We now define and analyze the parabolic space $L^{2}\left(0, T ; \dot{H}^{\frac{\alpha}{2}}\left(\mathbb{R}^{N}\right)\right)$. In the proof we will use the following iterated $L^{p}$-space [8]:

$L^{2}\left(0, T ; L^{q}\left(\mathbb{R}^{N}\right)\right)=\left\{f: \mathbb{R}^{N} \times(0, T) \rightarrow \mathbb{R}\right.$ measurable $\left.\mid \int_{0}^{T}\|f(\cdot, t)\|_{L^{q}}^{2} \mathrm{~d} t<\infty\right\}$,

for some $q \in(1, \infty)$. Note that this space is not a priori a Bochner space.

Lemma B.2. Let $\alpha \in(0,2), N>\alpha$, and $\mu_{\alpha}(\mathrm{d} z):=\frac{c_{N, \alpha} \mathrm{d} z}{|z|^{N+\alpha}}$. Then the space

$$
L^{2}\left(0, T ; \dot{H}^{\frac{\alpha}{2}}\left(\mathbb{R}^{N}\right)\right):=L^{2}\left(0, T ; L^{\frac{2 N}{N-\alpha}}\left(\mathbb{R}^{N}\right)\right) \cap L^{2}\left(0, T ; E_{\mu_{\alpha}}\left(\mathbb{R}^{N}\right)\right)
$$

is a Hilbert space with inner product

$$
\langle\psi, \phi\rangle:=\int_{0}^{T} \int_{\mathbb{R}^{N}}|\xi|^{\alpha} \mathcal{F}\{\psi(\cdot, t)\}(\xi) \overline{\mathcal{F}\{\phi(\cdot, t)\}(\xi)} \mathrm{d} \xi \mathrm{d} t=c_{N, \alpha} \int_{0}^{T} \mathcal{E}_{\mu_{\alpha}}[\psi, \phi] \mathrm{d} t .
$$

Moreover, $L^{2}\left(0, T ; \dot{H}^{\frac{\alpha}{2}}\left(\mathbb{R}^{N}\right)\right)={\overline{C_{\mathrm{c}}^{\infty}\left(\mathbb{R}^{N} \times[0, T)\right)}}^{\sqrt{\langle\cdot, \cdot\rangle}}$. 
Proof. 1) Embedding. Since $f \in L^{2}\left(0, T ; \dot{H}^{\frac{\alpha}{2}}\left(\mathbb{R}^{N}\right)\right)$, we have as a consequence of properties of iterated $L^{p}$-spaces $[8$ and Fubini's theorem that

$$
\|f(\cdot, t)\|_{L^{\frac{2 N}{N-\alpha}}\left(\mathbb{R}^{N}\right)} \in L^{2}(0, T) \quad \text { and } \quad|f(\cdot, t)|_{\dot{H}^{\frac{\alpha}{2}}\left(\mathbb{R}^{N}\right)} \in L^{2}(0, T) .
$$

It follows that for a.e. $t \in(0, T), f(\cdot, t) \in L^{\frac{2 N}{N-\alpha}}\left(\mathbb{R}^{N}\right) \cap E_{\mu_{\alpha}}\left(\mathbb{R}^{N}\right)$, and then $f(\cdot, t) \in \dot{H}_{1}^{\frac{\alpha}{2}}\left(\mathbb{R}^{N}\right)$ by Proposition B.1 (c). By (B.1) , we can then conclude that

$$
\int_{0}^{T}\|f(\cdot, t)\|_{L^{\frac{2 N}{N-\alpha}}\left(\mathbb{R}^{N}\right)}^{2} \mathrm{~d} t \leq C \int_{0}^{T}|f(\cdot, t)|_{\dot{H}^{\frac{\alpha}{2}}\left(\mathbb{R}^{N}\right)}^{2} \mathrm{~d} t .
$$

2) Inner product space. Obviously $\langle\cdot, \cdot\rangle=\int_{0}^{T}|\cdot|_{\dot{H}^{\frac{\alpha}{2}}\left(\mathbb{R}^{N}\right)}^{2} \mathrm{~d} t=|\cdot|_{T, E_{\mu_{\alpha}}}^{2}$ defines (the square of) a seminorm. By (‥4 $), \int_{0}^{T}|f(\cdot, t)|_{\dot{H}^{\frac{\alpha}{2}}\left(\mathbb{R}^{N}\right)}^{2} \mathrm{~d} t=0$ implies $f=0$ a.e. in $Q_{T}$, and hence the seminorm is a full norm. Now it is easy to check that the space is an inner product space.

3) Completeness. Let $\left\{f_{n}\right\}_{n \in \mathbb{N}}$ be a Cauchy sequence in $L^{2}\left(0, T ; \dot{H}^{\frac{\alpha}{2}}\left(\mathbb{R}^{N}\right)\right)$. By definition and (B.4), it follows that

$$
\int_{0}^{T}\left\|f_{n}(\cdot, t)-f_{m}(\cdot, t)\right\|_{L^{\frac{2 N}{N-\alpha}}\left(\mathbb{R}^{N}\right)}^{2} \mathrm{~d} t \leq \int_{0}^{T}\left|f_{n}(\cdot, t)-f_{m}(\cdot, t)\right|_{\dot{H}^{\frac{\alpha}{2}}\left(\mathbb{R}^{N}\right)}^{2} \mathrm{~d} t \rightarrow 0
$$

as $n, m \rightarrow \infty$. Hence the sequence is Cauchy also in $L^{2}\left(0, T ; L^{\frac{2 N}{N-\alpha}}\left(\mathbb{R}^{N}\right)\right)$. By [8], this space is complete, and sequences converging in norm contain pointwise a.e. converging subsequences. Therefore there is $f \in L^{2}\left(0, T ; L^{\frac{2 N}{N-\alpha}}\left(\mathbb{R}^{N}\right)\right)$ such that

$$
\int_{0}^{T}\left\|f_{n}(\cdot, t)-f(\cdot, t)\right\|_{L^{\frac{2 N}{N-\alpha}}\left(\mathbb{R}^{N}\right)} \rightarrow 0 \quad \text { as } \quad n \rightarrow \infty,
$$

and a subsequence $f_{n_{k}} \rightarrow f$ a.e. in $Q_{T}$ as $n \rightarrow \infty$. By Fatou's lemma,

$$
\begin{aligned}
\int_{0}^{T}\left|f_{m}(\cdot, t)-f(\cdot, t)\right|_{\dot{H}^{\frac{\alpha}{2}\left(\mathbb{R}^{N}\right)}}^{2} \mathrm{~d} t & =\int_{0}^{T}\left|f_{m}(\cdot, t)-\lim _{k \rightarrow \infty} f_{n_{k}}(\cdot, t)\right|_{\dot{H}^{\frac{\alpha}{2}}}^{2}\left(\mathbb{R}^{N}\right) \\
& \leq \liminf _{k \rightarrow \infty} \int_{0}^{T}\left|f_{m}(\cdot, t)-f_{n_{k}}(\cdot, t)\right|_{\dot{H}^{\frac{\alpha}{2}}\left(\mathbb{R}^{N}\right)}^{2} \mathrm{~d} t
\end{aligned}
$$

which goes to zero as $m \rightarrow \infty$. Hence $f_{n} \rightarrow f$ in $L^{2}\left(0, T ; \dot{H}^{\frac{\alpha}{2}}\left(\mathbb{R}^{N}\right)\right)$, and then by the triangle inequality $f \in L^{2}\left(0, T ; \dot{H}^{\frac{\alpha}{2}}\left(\mathbb{R}^{N}\right)\right)$.

4) Density. For any $f \in L^{2}\left(0, T ; \dot{H}^{\frac{\alpha}{2}}\left(\mathbb{R}^{N}\right)\right)$, the $C_{\mathrm{c}}^{\infty}\left(\mathbb{R}^{N} \times[0, T)\right)$ functions $w_{\delta}$ defined in (A.3) in Appendix A satisfy

$$
\left.\left\|w_{\delta}-f\right\|_{L^{2}(0, T ; L} \frac{2 N}{N-\alpha}\left(\mathbb{R}^{N}\right)\right) \stackrel{\delta \rightarrow 0^{+}}{\longrightarrow} 0 \quad \text { and } \quad\left|w_{\delta}\right|_{T, E_{\mu_{\alpha}}} \leq C|f|_{T, E_{\mu_{\alpha}}} .
$$


This follows from Remark A.4 and the fact that the iterated $L^{p}$-spaces have similar properties as the usual $L^{p}$-spaces with respect to mollifications (by [8], inequalities for convolutions, continuity of translations, dominated convergence etc. are similar). Since $L^{2}\left(0, T ; \dot{H}^{\frac{\alpha}{2}}\left(\mathbb{R}^{N}\right)\right)$ is a Hilbert space, Banach-Saks' theorem implies there is a subsequence $\left\{w_{\delta_{k}}\right\}_{k \in \mathbb{N}}$ and $\tilde{f} \in L^{2}\left(0, T ; \dot{H}^{\frac{\alpha}{2}}\left(\mathbb{R}^{N}\right)\right)$ such that

$$
\left|\frac{1}{n} \sum_{k=1}^{n} w_{\delta_{k}}-\tilde{f}\right|_{T, E_{\mu_{\alpha}}}^{2} \rightarrow 0 \quad \text { as } \quad n \rightarrow \infty .
$$

By (B.4), these Césaro means converge to $\tilde{f}$ in $L^{2}\left(0, T ; L^{\frac{2 N}{N-\alpha}}\left(\mathbb{R}^{N}\right)\right)$. But by $(\mathrm{B} .5)$, they also converge to $f$ in this space, and hence $f=\tilde{f}$ a.e.

\section{Proof of Theorem 2.6 (b)}

1) By $\left(\mathrm{A}_{\lambda 1}\right.$ "), the measure $\lambda$ is globally shift-bounded and the (semi)norms on $E_{\lambda}$ and $\dot{H}^{\frac{\alpha}{2}}$ are comparable: $m|f|_{\dot{H}^{\frac{\alpha}{2}}} \leq|f|_{E_{\lambda}} \leq M|f|_{\dot{H}^{\frac{\alpha}{2}}}$. The latter gives

$$
E_{\lambda}\left(\mathbb{R}^{N}\right)=\left\{f \text { is measurable }:|f|_{\dot{H}^{\frac{\alpha}{2}}}<\infty\right\}=E_{\mu_{\alpha}}\left(\mathbb{R}^{N}\right) .
$$

2) For $N \leq \alpha$, the fractional Laplacian (and hence also $A^{\lambda}$ ) is recurrent. In this case an easy modification of the proof of Theorem 3.1 in [36] yields

$$
X=L^{\infty}\left(Q_{T}\right) \cap L^{2}\left(0, T ; E_{\mu_{\alpha}}\left(\mathbb{R}^{N}\right)\right) .
$$

See the discussion on recurrence in Section 2.4 for more details.

$3)$ For $N>\alpha$, we always have $X \subset L^{\infty}\left(Q_{T}\right) \cap L^{2}\left(0, T ; E_{\mu_{\alpha}}\left(\mathbb{R}^{N}\right)\right)$. To prove the reverse inclusion, we must show that $C_{\mathrm{c}}^{\infty}$ is dense in $L^{\infty}\left(Q_{T}\right) \cap$ $L^{2}\left(0, T ; E_{\mu_{\alpha}}\left(\mathbb{R}^{N}\right)\right)$. This will follow from Lemma $\mathrm{B} .2$ if we can show that

$$
L^{\infty}\left(Q_{T}\right) \cap L^{2}\left(0, T ; E_{\mu_{\alpha}}\left(\mathbb{R}^{N}\right)\right) \subset L^{2}\left(0, T ; \dot{H}^{\frac{\alpha}{2}}\left(\mathbb{R}^{N}\right)\right) .
$$

To prove this, we must show that any $g \in L^{\infty}\left(Q_{T}\right) \cap L^{2}\left(0, T ; E_{\mu_{\alpha}}\left(\mathbb{R}^{N}\right)\right)$ also belongs to $L^{2}\left(0, T ; L^{\frac{2 N}{N-\alpha}}\left(\mathbb{R}^{N}\right)\right)$.

Let $h(t):=\|g(\cdot, t)\|_{L^{\frac{2 N}{N-\alpha}}\left(\mathbb{R}^{N}\right)}^{2}$ for $t \in(0, T)$. By Section 252P in 28] and measurability of $g$ on $\mathbb{R}^{N} \times(0, T), h$ is a measurable function on $(0, T)$. We need to prove that $h$ belongs to $L^{1}(0, T)$. As a consequence of Fubini's theorem,

$$
\|g(\cdot, t)\|_{L^{\infty}\left(\mathbb{R}^{N}\right)} \in L^{\infty}(0, T) \quad \text { and } \quad|g(\cdot, t)|_{\dot{H}^{\frac{\alpha}{2}}\left(\mathbb{R}^{N}\right)} \in L^{2}(0, T),
$$

and then $g(\cdot, t) \in L^{\infty}\left(\mathbb{R}^{N}\right) \cap E_{\mu_{\alpha}}\left(\mathbb{R}^{N}\right)$ for a.e. $t \in(0, T)$. Hence for such $t, g(\cdot, t)$ is a tempered distribution and belongs to $\dot{H}_{1}^{\frac{\alpha}{2}}\left(\mathbb{R}^{N}\right)$ by the argument of Step 4) in the proof of Proposition B.1. Then by the embedding (B.1), $h(t) \leq C|g(\cdot, t)|_{\dot{H}^{\frac{\alpha}{2}}\left(\mathbb{R}^{N}\right)}^{2}$ for a.e. $t$, and hence since $|g(\cdot, t)|_{\dot{H}^{\frac{\alpha}{2}}\left(\mathbb{R}^{N}\right)}^{2} \in L^{1}(0, T)$, it follows that $h \in L^{1}(0, T)$ and $g \in L^{2}\left(0, T ; L^{\frac{2 N}{N-\alpha}}\left(\mathbb{R}^{N}\right)\right)$. The proof is complete. 


\section{Proof of Lemma 4.3}

1) Assume $1<p, q<\infty$. Consider $f_{n}(x):=\left(f * \omega_{n}\right)(x)$ and $g_{n}(x):=$ $\left(g * \omega_{n}\right)(x)$ for $\omega_{n}$ defined by (4.1). By a direct computation,

$$
\begin{aligned}
& \left(f_{n} g_{n}\right)(x+z)-\left(f_{n} g_{n}\right)(x)-z \cdot D\left(f_{n} g_{n}\right)(x) \\
& =f_{n}(x)\left(g_{n}(x+z)-g_{n}(x)-z \cdot D g_{n}(x)\right) \\
& \quad+g_{n}(x)\left(f_{n}(x+z)-f_{n}(x)-z \cdot D f_{n}(x)\right) \\
& \quad+\left(f_{n}(x+z)-f_{n}(x)\right)\left(g_{n}(x+z)-g_{n}(x)\right)
\end{aligned}
$$

Integrate the above equality against $\nu(\mathrm{d} z) \mathrm{d} x$ to get

$$
\begin{aligned}
& \int_{\mathbb{R}^{N}} \mathcal{L}^{\nu}\left[f_{n} g_{n}\right](x) \mathrm{d} x \\
& =\int_{\mathbb{R}^{N}} f_{n}(x) \mathcal{L}^{\nu}\left[g_{n}\right](x) \mathrm{d} x+\int_{\mathbb{R}^{N}} g_{n}(x) \mathcal{L}^{\nu}\left[f_{n}\right](x) \mathrm{d} x+2 \mathcal{E}_{\nu}\left[f_{n}, g_{n}\right] .
\end{aligned}
$$

The three terms on the right-hand side are well-defined by Hölder's inequality since the measure $\nu$ is finite. By Fubini's theorem, $\int_{\mathbb{R}^{N}} \mathcal{L}^{\nu}\left[f_{n} g_{n}\right](x) \mathrm{d} x=0$ and thus, we obtain

$$
0=\int_{\mathbb{R}^{N}} f_{n}(x) \mathcal{L}^{\nu}\left[g_{n}\right](x) \mathrm{d} x+\int_{\mathbb{R}^{N}} g_{n}(x) \mathcal{L}^{\nu}\left[f_{n}\right](x) \mathrm{d} x+2 \mathcal{E}_{\nu}\left[f_{n}, g_{n}\right] .
$$

By standard estimates for mollifiers, Tonelli's lemma, and Hölder's inequality,

$$
\begin{aligned}
& \left|\int_{\mathbb{R}^{N}} f_{n} \mathcal{L}^{\nu}\left[g_{n}\right] \mathrm{d} x-\int_{\mathbb{R}^{N}} f \mathcal{L}^{\nu}[g] \mathrm{d} x\right| \\
& \leq 2 \nu\left(\mathbb{R}^{N}\right)\left(\|g\|_{L^{q}\left(\mathbb{R}^{N}\right)}\left\|f_{n}-f\right\|_{L^{p}\left(\mathbb{R}^{N}\right)}+\|f\|_{L^{p}\left(\mathbb{R}^{N}\right)}\left\|g_{n}-g\right\|_{L^{q}\left(\mathbb{R}^{N}\right)},\right.
\end{aligned}
$$

and

$$
\begin{aligned}
& \left|2 \mathcal{E}_{\nu}\left[f_{n}, g_{n}\right]-2 \mathcal{E}_{\nu}[f, g]\right| \\
& \leq 4 \nu\left(\mathbb{R}^{N}\right)\left(\|g\|_{L^{q}\left(\mathbb{R}^{N}\right)}\left\|f_{n}-f\right\|_{L^{p}\left(\mathbb{R}^{N}\right)}+\|f\|_{L^{p}\left(\mathbb{R}^{N}\right)}\left\|g_{n}-g\right\|_{L^{q}\left(\mathbb{R}^{N}\right)}\right) .
\end{aligned}
$$

Note that a similar argument holds for $\int_{\mathbb{R}^{N}} g_{n} \mathcal{L}^{\nu}\left[f_{n}\right] \mathrm{d} x$. Taking the limit as $n \rightarrow \infty$ and using the properties of mollifiers, we obtain (D.1) for $f, g$ replacing $f_{n}, g_{n}$ respectively. Since $\mathcal{L}^{\nu}$ is symmetric, we obtain

$$
\int_{\mathbb{R}^{N}} g(x) \mathcal{L}^{\nu}[f](x) \mathrm{d} x=-\mathcal{E}_{\nu}[f, g] .
$$

2) Assume $p=1, q=\infty$. Again we mollify, $f_{n}(x):=\left(f * \omega_{n}\right)(x)$ and $g_{m}(x):=\left(g * \omega_{m}\right)(x)$, and we obtain (D.1) as above. We deduce (almost as 
before) that

$$
\begin{aligned}
& \left|\int_{\mathbb{R}^{N}} f_{n} \mathcal{L}^{\nu}\left[g_{m}\right] \mathrm{d} x-\int_{\mathbb{R}^{N}} f \mathcal{L}^{\nu}[g] \mathrm{d} x\right| \\
& \leq 2 \nu\left(\mathbb{R}^{N}\right)\|g\|_{L^{\infty}\left(\mathbb{R}^{N}\right)}\left\|f_{n}-f\right\|_{L^{1}\left(\mathbb{R}^{N}\right)} \\
& \quad+\int_{\mathbb{R}^{N}} \int_{\mathbb{R}^{N}}\left|f_{n}(x)\right|\left|\left(g_{m}(x+z)-g(x+z)\right)-\left(g_{m}(x)-g(x)\right)\right| \nu(\mathrm{d} z) \mathrm{d} x,
\end{aligned}
$$

and

$$
\begin{aligned}
\left|2 \mathcal{E}_{\nu}\left[f_{n}, g_{m}\right]-2 \mathcal{E}_{\nu}[f, g]\right| \leq & \int_{\mathbb{R}^{N}} \int_{\mathbb{R}^{N}}\left|f_{n}(x+z)-f_{n}(x)\right| \times \\
& \times\left|\left(g_{m}(x+z)-g(x+z)\right)-\left(g_{m}(x)-g(x)\right)\right| \nu(\mathrm{d} z) \mathrm{d} x \\
& +4 \nu\left(\mathbb{R}^{N}\right)\|g\|_{L^{\infty}\left(\mathbb{R}^{N}\right)}\left\|f_{n}-f\right\|_{L^{1}\left(\mathbb{R}^{N}\right)}
\end{aligned}
$$

Note that $\left|\left(g_{m}(x+z)-g(x+z)\right)-\left(g_{m}(x)-g(x)\right)\right| \leq 4\|g\|_{L^{\infty}\left(\mathbb{R}^{N}\right)}$ and $\left|f_{n}(x)\right| \in L^{1}\left(\mathbb{R}^{N}\right)$. Hence, for fixed $n$, we may send $m \rightarrow \infty$ by Lebesgue's dominated convergence theorem to obtain (D.1) for $f_{n}, g$. Then we send $n \rightarrow \infty$ to obtain the same for $f, g$. Again, we use the symmetry of $\mathcal{L}^{\nu}$ to complete the proof.

\section{References}

[1] N. Alibaud. Entropy formulation for fractal conservation laws. J. Evol. Equ., $7(1): 145-175,2007$.

[2] N. Alibaud and B. Andreianov. Non-uniqueness of weak solutions for the fractal Burgers equation. Ann. Inst. H. Poincaré Anal. Non Linéaire, 27(4):9971016, 2010.

[3] F. Andreu-Vaillo, J. M. Mazon, J. D. Rossi and J. J. Toledo-Melero. Nonlocal Diffusion Problems Math. Surveys Monogr., 165, AMS, Rhode Island, 2010.

[4] D. Applebaum. Lévy processes and Stochastic Calculus. Cambridge Studies in Advanced Mathematics, 116, Cambridge University Press, Cambridge, 2009.

[5] M. T. Barlow, R. F. Bass, Z.-Q. Chen and M. Kassmann. Non-local Dirichlet forms and symmetric jump processes. Trans. Amer. Math. Soc., 361(4):19631999, 2009.

[6] B. Barrios, I. Peral, F. Soria and E. Valdinoci. A Widder's type theorem for the heat equation with nonlocal diffusion. Arch. Ration. Mech. Anal., 213(2):629-650, 2014.

[7] H. Bahouri, J.-Y. Chemin And R. Danchin. Fourier Analysis and Nonlinear Partial Differential Equations. Grundlehren der mathematischen Wissenschaften, 343, Springer-Verlag, Berlin-Heidelberg, 2001. 
[8] A. Benedek and R. Panzone. The space $L^{p}$, with mixed norm. Duke Math. J., 28(3):301-324, 1961.

[9] P. Biler, C. Imbert And G. Karch. The nonlocal porous medium equation: Barenblatt profiles and other weak solutions. Arch. Ration. Mech. Anal., 215:497529, 2015.

[10] P. Biler, G. Karch and R. Monneau. Nonlinear diffusion of dislocation density and self-similar solutions. Comm. Math. Phys., 294(1):145-168, 2010.

[11] M. Bonforte, A. Segatti and J. L. VÁzquez. Non-existence and instantaneous extinction of solutions for singular nonlinear fractional diffusion equations. Calc. Var. Partial Differential Equations, 55(3):55-68, 2016.

[12] M. Bonforte, Y. Sire And J. L. VAzQuez. Existence, Uniqueness and Asymptotic behaviour for fractional porous medium equations on bounded domains. Discrete Contin. Dyn. Syst., 35(12):5725-5767, 2015.

[13] M. Bonforte, Y. Sire And J. L. VÁzquez. Optimal Existence and Uniqueness Theory for the Fractional Heat Equation. Nonlinear Anal. TMA., 153:142-168, 2017.

[14] M. Bonforte And J. L. VÁzquez. Quantitative Local and Global A Priori Estimates for Fractional Nonlinear Diffusion Equations. Adv. Math., 250:242$284,2014$.

[15] M. Bonforte And J. L. VÁzquez. A Priori Estimates for Fractional Nonlinear Degenerate Diffusion Equations on bounded domains. Arch. Ration. Mech. Anal., 218(1):317-362, 2015.

[16] M. Bonforte And J. L. VÁzquez. Fractional Nonlinear Degenerate Diffusion Equations on Bounded Domains Part I. Existence, Uniqueness and Upper Bounds. Nonlinear Anal. TMA., 131:363-398, 2016.

[17] C. Brändle And A. DE PABlo. Nonlocal heat equations: decay estimates and Nash inequalities. Preprint, arXiv:1312.4661v4 [math.AP], 2015.

[18] H. Brézis and M. G. CRAndall. Uniqueness of solutions of the initial-value problem for $u_{t}-\triangle \varphi(u)=0$. J. Math. Pures Appl., 58(2):153-163, 1979.

[19] L. Caffarelli And J. L. VazQuez. Nonlinear porous medium flow with fractional potential pressure. Arch. Ration. Mech. Anal., 202(2):537-565, 2011.

[20] S. Cifani And E. R. Jakobsen. Entropy formulation for degenerate fractional order convection-diffusion equations. Ann. Inst. H. Poincaré Anal. Non Linéaire, 28(3):413-441, 2011.

[21] J. Endal and E. R. Jakobsen. $L^{1}$ Contraction for Bounded (Nonintegrable) Solutions of Degenerate Parabolic Equations. SIAM J. Math. Anal., 46(6):39573982, 2014.

[22] F. Del Teso, J. Endal and E. R. Jakobsen. Uniqueness and properties of distributional solutions of nonlocal equations of porous medium type. $A d v$. Math., 305:78-143, 2017.

[23] F. Del Teso, J. Endal and E. R. Jakobsen. Numerical analysis and methods for distributional solutions of nonlocal (and local) equations of porous medium type. In preparation, 2016. 
[24] A. De Pablo, F. Quirós And A. Rodríguez. Nonlocal filtration equations with rough kernels. Nonlinear Anal. TMA, 137:402-425, 2016.

[25] A. De Pablo, F. Quirós, A. Rodríguez and J. L. VÁzquez. A fractional porous medium equation. Adv. Math., 226(2):1378-1409, 2011.

[26] A. De Pablo, F. Quirós, A. Rodríguez and J. L. Vázquez. A general fractional porous medium equation. Comm. Pure Appl. Math., 65(9):1242-1284, 2012.

[27] A. de Pablo, F. Quirós, A. Rodríguez and J. L. VÁzquez. Classical solutions for a logarithmic fractional diffusion equation. J. Math. Pures Appl., 101(6):901-924, 2014.

[28] D. H. Fremlin. Measure theory. Vol. 2: Broad Foundations. Torres Fremlin, Colchester, 2001.

[29] M. Fukushima, Y. Oshima, and M. Takeda. Dirichlet Forms and Symmetric Markov Processes. Studies in Mathematics, 19, De Gruyter, Berlin, 1994.

[30] L. Grillo, M. Muratori And F. Punzo. Fractional porous media equations: existence and uniqueness of weak solutions with measure data. Calc. Var. Partial Differential Equations, 54(3):3303-3335, 2015.

[31] M. Kassmann and R. W. Schwab. Regularity results for nonlocal parabolic equations. Riv. Mat. Univ. Parma, 5(1):183-212, 2014.

[32] O. A. Olě́nik, A. S. Kalašnikov and Y.-I. Čžou. The Cauchy problem and boundary problems for equations of the type of non-stationary filtration. (In Russian) Izv. Akad. Nauk SSSR. Ser. Mat., 22:667-704, 1958.

[33] G. Palatucci And A. Pisante. Improved Sobolev embeddings, profile decomposition, and concentration-compactness for fractional Sobolev spaces. Calc. Var., 50:799-829, 2014.

[34] K. Sato. Lévy processes and infinitely divisible distributions. Cambridge Studies in Advanced Mathematics, 68, Cambridge University Press, Cambridge, 1999.

[35] R. L. Schilling And T. Uemura. On the Feller property of Dirichlet forms generated by pseudo differential operators. Tôhoku Math. J., 59:401-422, 2007.

[36] R. L. Schilling and T. Uemura. On the Structure of the Domain of a Symmetric Jump-type Dirichlet Form. Publ. RIMS Kyoto Univ., 48:1-20, 2012.

[37] L. Silvestre. Regularity estimates for parabolic integro-differential equations and applications. In: Proceedings of the International Congress of Mathematicians, vol. III, 873-894, Kyung Moon SA, Seoul, 2014.

[38] D. Stan, F. Del Teso and J. L. VÁzquez. Finite and infinite speed of propagation for porous medium equations with fractional pressure. C. R. Math. Acad. Sci. Paris, 119:62-73, 2014.

[39] D. Stan, F. Del Teso and J. L. VÁzquez. Transformations of self-similar solutions for porous medium equations of fractional type. Nonlinear Anal. TMA, 119:62-73, 2015.

[40] D. Stan, F. Del Teso and J. L. VÁzquez. Finite and infinite speed of propagation for porous medium equations with nonlocal pressure. J. Differential Equations, 260(2): 1154-1199, 2016. 
[41] D. W. Stroock and S. R. S. Varadhan. Multidimensional diffusion processes. Grundlehren der mathematischen Wissenschaften, 233, Springer-Verlag, BerlinNew York, 1979.

[42] J. L. VÁZQUEZ. Smoothing and decay estimates for nonlinear diffusion equations. Equations of porous medium type. Oxford Lecture Series in Mathematics and its Applications, 33, Oxford University Press, Oxford, 2006.

[43] J. L. VÁzQuez. The porous medium equation. Mathematical theory. Oxford Math. Monogr., The Clarendon Press, Oxford University Press, Oxford, 2007.

[44] W. P. Ziemer. Weakly Differentiable functions: Sobolev Spaces and Functions of Bounded Variation. Graduate texts in mathematics, 120, Springer-Verlag, New York, 1989.

Department of Mathematical Sciences

Norwegian University of Science and Technology (NTNU)

N-7491 Trondheim, Norway

E-mail: felix.delteso@ntnu.no, jorgen.endal@ntnu.no, espen.jakobsen@ntnu.no 